\title{
Odd primary homotopy decompositions of gauge groups
}

\author{
STEPHEN D THERIAULT
}

\begin{abstract}
We construct $p$-local decompositions of certain gauge groups when $p$ is an odd prime. Specifically, we decompose $\mathrm{SU}(n), \mathrm{Sp}(n)$ and $\mathrm{Spin}(n)$-gauge groups over simply connected 4-manifolds and $U(n)$-gauge groups over compact, orientable Riemann surfaces, given certain restrictions on $n$ that depend on $p$.
\end{abstract}

$54 \mathrm{C} 35,55 \mathrm{P} 35,55 \mathrm{R} 10$

\section{Introduction}

Let $X$ be a path-connected, pointed topological space and $G$ a topological group with classifying space $B G$. Let $P \longrightarrow X$ be a principal $G$-bundle. The gauge group $\mathcal{G}(P)$ is the group of $G$-equivariant automorphisms of $P$ that fix $X$. The topology of gauge groups and their classifying spaces plays a crucial role in mathematical physics and the geometry of 4-manifolds. In physics, a gauge group describes the parallel transport of point particles by means of connections on bundles. The role that the topology of gauge groups plays is nicely described by Cohen and Milgram [5]. In geometry, Donaldson [8] famously used topological information from SU(2)-gauge groups to help identify polynomial invariants that distinguished between diffeomorphism structures on 4-dimensional Riemannian manifolds. Topological properties of gauge groups have received considerable attention over the past twenty years, particularly in terms of counting their distinct homotopy types (see Crabb and Sutherland [7], Hamanaka and Kono [14], Kono [19], Kono and Tsukuda [20] and Sutherland [25]) and calculating the homology and cohomology of their classifying spaces (see Crabb [6], Masbaum [22] and Terzić [26]).

In this paper we consider how certain gauge groups decompose as products after localization at an odd prime $p$. The goal is to produce analogues of the $p$-local decompositions of simple, compact Lie groups determined by Harris [15], Mimura and Toda [24] and Mimura, Nishida and Toda [23], which have proved to be useful when investigating the homotopy theory of Lie groups. It is expected that the decompositions proved here will be equally useful when investigating the homotopy theory of gauge groups. 
We consider two collections of gauge groups. The first often arises in mathematical physics, and is associated to principal $G$-bundles over a simply connected 4manifold $M$, where $G$ is a simple, simply connected compact Lie group. The second is associated to principal $U(n)$-bundles over a compact, orientable Riemann surface $\Sigma_{g}$ of genus $g$. Since the set of homotopy classes $[M, B G]$ is isomorphic to $\mathbb{Z}$, each integer $k$ classifies up to isomorphism a principal $G$-bundle $P_{k}$ over $M$. Let $\mathcal{G}_{k} G$ be the gauge group of $P_{k}$. Similarly, as $\left[\Sigma_{g}, B U(n)\right] \cong \mathbb{Z}$, to each integer $k$ there is an isomorphism class of principal $U(n)$-bundles over $\Sigma_{g}$ with gauge group $\mathcal{G}_{k} U(n)$.

We begin with a straightforward decomposition in Section 2 to reduce to the case when the 4-manifold is $S^{4}$, or when the Riemann surface is $S^{2}$. To analyze these, we use Gottlieb's general result [11] (see also Atiyah and Bott [1]) that for any topological group $G$ and path-connected space $X$, the gauge group $\mathcal{G}(P)$ of a principal $G$-bundle has classifying space $B \mathcal{G}(P)$ homotopy equivalent to $\operatorname{Map}_{P}(X, B G)$, the space of continuous maps from $X$ to $B G$ that induce $P$. Evaluating at the basepoint of $X$ yields a fibration $\operatorname{Map}_{P}^{*}(X, B G) \longrightarrow \operatorname{Map}_{P}(X, B G) \longrightarrow B G$, where the fiber is the space of continuous, pointed maps from $X$ to $B G$ that induce $P$. This fibration has a connecting map $\partial_{k}: G \rightarrow \operatorname{Map}_{P}^{*}(X, B G)$. For any integer $m$ and space $Y$, let $\Omega_{0}^{m} Y$ denote the component of $\Omega^{m} Y$ containing the basepoint. Equivalently, if $Y\langle m\rangle$ is the $m$-connected cover of $Y$, then $\Omega_{0}^{m} Y \simeq \Omega^{m} Y\langle m\rangle$. In our cases, it is well known (see Sutherland [25] for example) that the components $\operatorname{Map}_{k}^{*}\left(S^{4}, B G\right)$ are all homotopy equivalent to $\Omega_{0}^{3} G$, and the components $\operatorname{Map}_{k}^{*}\left(S^{2}, B U(n)\right)$ are all homotopy equivalent to $\Omega_{0} U(n) \simeq \Omega \mathrm{SU}(n)$. So the connecting map $\partial_{k}$ induces homotopy fibrations

$$
\mathcal{G}_{k} G \longrightarrow G \stackrel{\partial_{k}}{\longrightarrow} \Omega_{0}^{3} G \quad \text { and } \quad \mathcal{G}_{k} U(n) \longrightarrow U(n) \stackrel{\partial_{k}}{\longrightarrow} \Omega_{0} U(n) .
$$

Our aim is to factor the maps $\partial_{k}$ in a useful way in order to produce $p$-local decompositions of $\mathcal{G}_{k} G$ and $\mathcal{G}_{k} U(n)$. This is the key work in the paper, and should have applications elsewhere. Note that $p$-localization is valid in this context by Hilton, Mislin and Roitberg [16], as the spaces $G, \Omega_{0}^{3} G, U(n)$, and $\Omega_{0} U(n)$ all have a nilpotent (in fact abelian) fundamental group which acts nilpotently on the higher homotopy groups.

We begin with a general theorem which is the driving force behind our results. Assume that all spaces and maps have been localized at an odd prime $p$ and take homology with mod- $p$ coefficients. It is well known that if the integral homology of $G$ is $p$-torsion free then there is an isomorphism $H_{*}(G) \cong \Lambda\left(x_{2 n_{1}+1}, \ldots, x_{2 n_{l}+1}\right)$, where $n_{1}<n_{2}<$ $\cdots<n_{l}$, the degree of $x_{2 n_{i}+1}$ is $2 n_{i}+1$, and the type of $G$ is $\left\{2 n_{1}-1, \ldots, 2 n_{l}-1\right\}$. 
Let $q=2(p-1)$. It is also well known that there is an algebra decomposition

$$
H_{*}(G) \cong \bigotimes_{i=1}^{p-1} \Lambda\left(V_{i}\right)
$$

where $V_{i}$ consists of those generators in $\left\{x_{2 n_{1}+1}, \ldots, x_{2 n_{l}+1}\right\}$ whose degrees are of the form $2 i+j q+1$ for some $j \geq 0$. Note that, depending on $G$, it may be the case that $V_{i}=\varnothing$ for some $i$. Mimura, Nishida and Toda [23] realized this algebra decomposition geometrically by showing that there is a homotopy equivalence $G \simeq \prod_{i=1}^{p-1} \bar{B}_{i}$ where $H_{*}\left(\bar{B}_{i}\right) \cong \Lambda\left(V_{i}\right)$. An alternative equivalence $e: G \rightarrow \prod_{i=1}^{p-1} B_{i}$ with $H_{*}\left(B_{i}\right) \cong \Lambda\left(V_{i}\right)$ was given in [28] in low ranks in which the factors and the equivalence are better controlled. The list of groups and ranks for which this holds is as follows:

$$
\begin{array}{ll}
\operatorname{SU}(n) & \text { if } n \leq(p-1)(p-2)+1 \\
\operatorname{Sp}(n) & \text { if } 2 n \leq(p-1)(p-2) \\
\operatorname{Spin}(2 n+1) & \text { if } 2 n \leq(p-1)(p-2) \\
\operatorname{Spin}(2 n) & \text { if } 2(n-1) \leq(p-1)(p-2) \\
G_{2}, F_{4}, E_{6} & \text { if } p \geq 5 \\
E_{7}, E_{8} & \text { if } p \geq 7
\end{array}
$$

The restrictions on $n$ and $p$ come from the fact that in these cases $G$ is a retract of $\Omega \Sigma A$, where $A$ is an appropriate subcomplex of $G$ (more will be said on this in Section 3).

Theorem 1.1 If $G$ is one of the groups in (1) then there is a homotopy commutative diagram

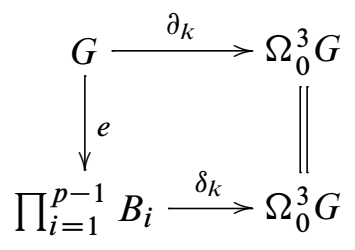

where $\delta_{k}$ is an $H$-map.

The key here is the combination of $\delta_{k}$ being an $H$-map and the fact that $G$ retracts off $\Omega \Sigma A$. This lets us deduce properties about $\delta_{k}$ by restricting to $A$. As one example, we have an analogue to the classical fact that if $G$ has type $\left\{2 n_{1}-1, \ldots, 2 n_{l}-1\right\}$ and $p \geq n_{l}$ then there is a homotopy decomposition $G \simeq \prod_{i=1}^{l} S^{2 n_{i}-1}$. 
Proposition 1.2 Suppose the type of $G$ is $\left\{2 n_{1}-1, \ldots, 2 n_{l}-1\right\}$. If $p>n_{l}+1$ then there is a homotopy equivalence

$$
\mathcal{G}_{k} G \simeq S^{3} \times \Omega^{4} S^{3}\langle 3\rangle \times \prod_{i=2}^{l}\left(S^{2 n_{i}-1} \times \Omega^{4} S^{2 n_{i}-1}\right) .
$$

In particular, Proposition 1.2 is a generalization to large primes of the rational decomposition of $\mathcal{G}_{k} G$ in Félix and Oprea [9].

The main application of Theorem 1.1 is to decompose $\mathcal{G}_{k} G$ when $G$ is one of $\operatorname{SU}(n)$, $\operatorname{Sp}(n)$ or $\operatorname{Spin}(n)$, as well as $\mathcal{G}_{k} U(n)$ for $n \leq(p-1)(p-2)+1$. We state the $\mathrm{SU}(n)$ case, deferring the others to the body of the paper. The homotopy equivalence $e$ takes the more explicit form

$$
\mathrm{SU}(n) \simeq \prod_{i=1}^{p-1} B_{i}
$$

where the homology of $B_{i}$ is $H_{*}\left(B_{i}\right) \cong \Lambda\left(x_{2 i+1}, x_{2 i+q+1}, \ldots, x_{2 i+\left(k_{n}-1\right) q+1}\right)$ and $k_{n}=\lfloor(n-i-1) /(p-1)\rfloor$ is the greatest integer less than or equal to $(n-i-1) /(p-1)$. Let $E: S^{m} \rightarrow \Omega S^{m+1}$ be the suspension. We show that the $H-$ map $\delta_{k}$ in Theorem 1.1 is homotopic to the composite

$$
\begin{aligned}
\prod_{i=1}^{p-1} B_{i} & \stackrel{\pi_{\mu, \nu}}{\longrightarrow} B_{\mu} \times B_{\nu} \longrightarrow S^{2 n-3} \times S^{2 n-1} \stackrel{E^{4} \times E^{4}}{\longrightarrow} \Omega^{4} S^{2 n+1} \times \Omega^{4} S^{2 n+3} \\
\stackrel{k \Omega^{3} \bar{s}_{n} \times k \Omega^{3} \bar{s}_{n+1}}{\longrightarrow} & \Omega_{0}^{3} B_{u} \times \Omega_{0}^{3} B_{v} \stackrel{i_{u, v}}{\longrightarrow} \prod_{i=1}^{p-1} \Omega_{0}^{3} B_{i} \stackrel{\Omega^{3} e^{-1}}{\longrightarrow} \Omega_{0}^{3} \operatorname{SU}(n) .
\end{aligned}
$$

Here, $\pi_{\mu, \nu}$ is the projection onto the factors $B_{\mu}$ and $B_{v}$ carrying the degree $2 n-3$ and $2 n-1$ generators in homology, which is followed by the maps to the corresponding spheres, $E^{4}$ is the quadruple suspension, $i_{u, v}$ is the inclusion of the factors $B_{u}$ and $B_{v}$ which inherit from the equivalence $e$ the homotopy groups $\pi_{2 n}(\mathrm{SU}(n)) \cong \mathbb{Z} / n ! \mathbb{Z}$ and $\pi_{2 n+2}(\mathrm{SU}(n)) \cong \mathbb{Z} /(n+1) ! \mathbb{Z}$ (a minor simplification of the latter has been incorporated which is valid at odd primes), and these generators are represented by the composites $\bar{s}_{n} \circ E$ and $\bar{s}_{n+1} \circ E$. The decomposition of $\mathcal{G}_{k} \mathrm{SU}(n)$ is a consequence of this factorization of $\delta_{k}$. Let $X_{k}$ and $Y_{k}$ respectively be the homotopy fibers of the composites

$$
\begin{aligned}
& B_{\mu} \longrightarrow S^{2 n-3} \stackrel{E^{4}}{\longrightarrow} \Omega^{4} S^{2 n+1} \stackrel{k \Omega^{3} \bar{s}_{n}}{\longrightarrow} \Omega_{0}^{3} B_{u} \\
& B_{v} \longrightarrow S^{2 n-1} \stackrel{E^{4}}{\longrightarrow} \Omega^{4} S^{2 n+3} \stackrel{k \Omega^{3} \bar{s}_{n+1}}{\longrightarrow} \Omega_{0}^{3} B_{v} .
\end{aligned}
$$


Theorem 1.3 Suppose $2 \leq n \leq(p-1)(p-2)+1$ and let $k \in \mathbb{Z}$. There is a homotopy decomposition

$$
\mathcal{G}_{k} \mathrm{SU}(n) \simeq X_{k} \times Y_{k} \times\left(\prod_{\substack{j=1 \\ j \neq \mu, v}}^{p-1} B_{j}\right) \times\left(\prod_{\substack{i=1 \\ i \neq u, v}}^{p-1} \Omega_{0}^{4} B_{i}\right)
$$

For example, if $n=p$ then by Mimura and Toda [24], $\mathrm{SU}(p) \simeq \prod_{i=1}^{p-1} S^{2 i-1}$. The map $\delta_{k}$ is homotopic to the composite

$$
\prod_{i=1}^{p-1} S^{2 i-1} \stackrel{\pi}{\longrightarrow} S^{2 p-3} \times S^{2 p-1} \stackrel{\alpha \times \alpha^{\prime}}{\longrightarrow} \Omega_{0}^{3} S^{3} \times \Omega^{3} S^{5} \stackrel{\imath}{\rightarrow} \prod_{i=1}^{p-1} \Omega_{0}^{3} S^{2 i-1} \stackrel{\Omega^{3} e^{-1}}{\longrightarrow} \Omega_{0}^{3} \operatorname{SU}(p),
$$

where $\pi$ is the projection, $\alpha$ and $\alpha^{\prime}$ represent the least nonvanishing torsion homotopy groups of $S^{3}$ and $S^{5}$ respectively - both of which have order $p$, and $\iota$ is the injection. Consequently, $\mathcal{G}_{k} \mathrm{SU}(p) \simeq X \times Y \times \prod_{j=1}^{p-3} S^{2 j-1} \times \prod_{i=1}^{p-1} \Omega^{4} S^{2 i-1}$, where $X$ and $Y$ are the homotopy fibres of $\alpha$ and $\alpha^{\prime}$ respectively.

Analogous decompositions of $\mathcal{G}_{k} \operatorname{Sp}(n), \mathcal{G}_{k} \operatorname{Spin}(2 n)$ and $\mathcal{G}_{k} \operatorname{Spin}(2 n+1)$ are given in Theorems 6.2, 7.4 and 7.1 respectively, as well as a decomposition of $\mathcal{G}_{k} U(n)$ in Theorem 6.3.

This paper is organized as follows. In Section 2 we reduce from simply connected 4-manifolds to $S^{4}$ and compact, orientable Riemann surfaces to $S^{2}$. In Section 3 we describe the properties we need of the low rank decomposition of $G$. Theorem 1.1 is proved in Section 4, and several consequences are given. In Section 5 we specialize to the cases when $G=\mathrm{SU}(n)$ and $G=\mathrm{Sp}(n)$, as well as consider the $U(n)$ case, and prove factorizations of restricted versions of $\partial_{k}$. These are then combined with Theorem 1.1 in Section 6 to factor $\partial_{k}$ itself. The decomposition in Theorem 1.3 follows, as well as analogues for $\mathcal{G}_{k} \operatorname{Sp}(n)$ and $\mathcal{G}_{k} U(n)$. Finally, in Section 7 we prove general results which decompose $\mathcal{G}_{k} \operatorname{Spin}(2 n+1)$ and $\mathcal{G}_{k} \operatorname{Spin}(2 n)$ in terms of $\mathcal{G}_{k} \operatorname{Sp}(m)$ for appropriate values of $m$.

The author would like to thank the referee for a careful reading of the paper, and for pointing out an omission in an earlier version of Theorem 7.1.

\section{Reduction to the case when $M=S^{4}$}

This section reduces the problem of decomposing gauge groups of principal $G$-bundles over simply connected 4 -manifolds to the special case when the manifold is $S^{4}$, and of principal $U(n)$-bundles over compact, orientable Riemann surfaces to the special case of $S^{2}$. 
First consider the case of a simply connected Spin 4-manifold $M$. Recall that $M$ has a CW-structure with has one 0 -cell, $d$ 2-cells for some nonnegative integer $d$, and one 4-cell. This CW-structure implies that there is a homotopy cofibration sequence

$$
S^{3} \stackrel{f}{\longrightarrow} \bigvee_{i=1}^{d} S^{2} \stackrel{i}{\longrightarrow} M \stackrel{q}{\longrightarrow} S^{4}
$$

where $f$ attaches the top cell, $i$ is the inclusion, and $q$ pinches onto the top cell. Since $M$ is a Spin-manifold, $\Sigma f$ is null homotopic. Thus there is a homotopy decomposition $\Sigma M \simeq\left(\bigvee_{i=1}^{d} S^{3}\right) \vee S^{5}$.

Consider the $k$-th component of the evaluation fibration

$$
\operatorname{Map}_{k}^{*}(M, B G) \longrightarrow \operatorname{Map}_{k}(M, B G) \stackrel{\mathrm{ev}}{\longrightarrow} B G .
$$

The evaluation map is natural so the pinch map $M \stackrel{q}{\longrightarrow} S^{4}$ determines a homotopy fibration diagram

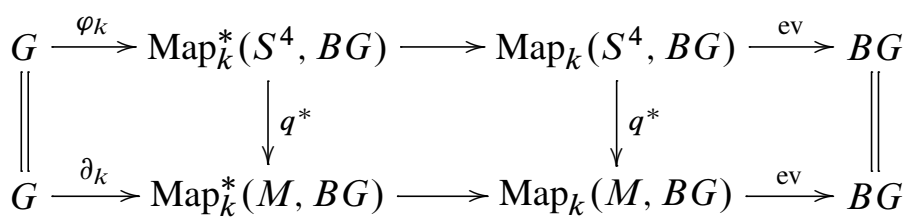

which defines the map $\varphi_{k}$. To distinguish between the associated gauge groups, let $\mathcal{G}_{k}(M, G)=\Omega \operatorname{Map}_{k}(M, B G)$ and $\mathcal{G}_{k} G=\Omega \operatorname{Map}_{k}\left(S^{4}, B G\right)$. The following decomposition is essentially a consequence of the triviality of $\Sigma f$.

Proposition 2.1 If $M$ is a simply connected Spin 4-manifold with $d$ two-cells then there is an integral homotopy decomposition

$$
\mathcal{G}_{k}(M, G) \simeq\left(\prod_{i=1}^{d} \Omega^{2} G\right) \times \mathcal{G}_{k} G .
$$

Proof The cofibration sequence

$$
M \stackrel{q}{\longrightarrow} S^{4} \stackrel{\Sigma f}{\longrightarrow} \bigvee_{i=1}^{d} S^{3} \stackrel{\Sigma i}{\longrightarrow} \Sigma M
$$


induces a fibration sequence

$\operatorname{Map}_{k}^{*}(\Sigma M, B G) \stackrel{(\Sigma i)^{*}}{\longrightarrow} \operatorname{Map}_{k}^{*}\left(\bigvee_{i=1}^{d} S^{3}, B G\right) \stackrel{(\Sigma f)^{*}}{\longrightarrow} \operatorname{Map}_{k}^{*}\left(S^{4}, B G\right)$

$\stackrel{q^{*}}{\longrightarrow} \operatorname{Map}_{k}^{*}(M, B G)$.

The factorization of $\partial_{k}$ through $\varphi_{k}$ in (2) implies that there is a homotopy commutative diagram

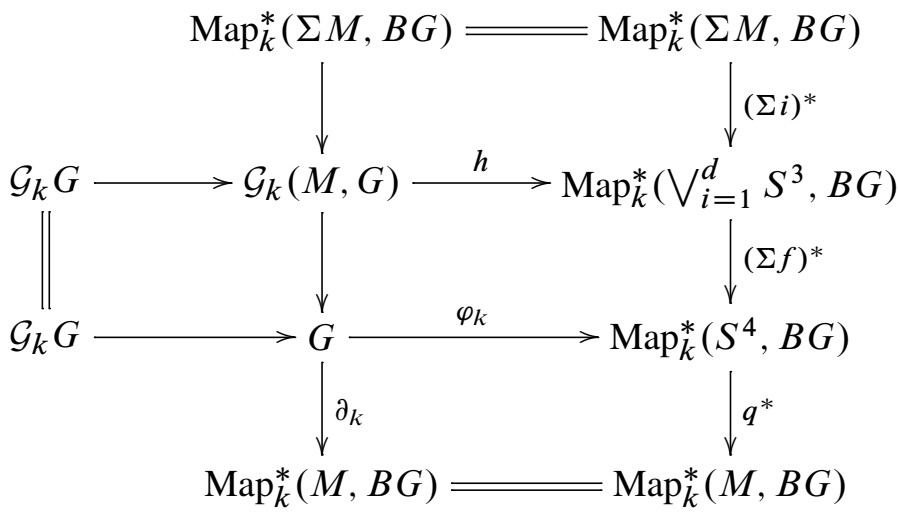

which defines the map $h$. Focus on the fibration in the second row, in which $h$ is the map to the base. Note that $\operatorname{Map}_{k}^{*}\left(\bigvee_{i=1}^{d} S^{3}, B G\right) \simeq \prod_{i=1}^{d} \Omega^{2} G$. Since $\Sigma f$ is null homotopic, so is $(\Sigma f)^{*}$. Thus $(\Sigma i)^{*}$ has a right homotopy inverse. This implies that $h$ has a right homotopy inverse, and so the loop structure on $\mathcal{G}_{k}(M, G)$ lets us multiply to obtain a homotopy equivalence

$$
\left(\prod_{i=1}^{d} \Omega^{2} G\right) \times \mathcal{G}_{k} G \longrightarrow \mathcal{G}_{k}(M, G) \times \mathcal{G}_{k}(M, G) \longrightarrow \mathcal{G}_{k}(M, G) .
$$

For a non-Spin-manifold, again there is a homotopy cofibration

$$
S^{3} \stackrel{f}{\longrightarrow} \bigvee_{i=1}^{d} S^{2} \stackrel{i}{\longrightarrow} M \stackrel{q}{\longrightarrow} S^{4}
$$

but now $\Sigma f$ is not null homotopic. Since $\pi_{4}\left(S^{3}\right)=\mathbb{Z} / 2 \mathbb{Z}$ it follows that $\Sigma f$ has order 2. So if we localize away from 2 then $\Sigma f$ is null homotopic and there is a $\mathbb{Z}_{(1 / 2)}$-local decomposition $\Sigma M \simeq\left(\bigvee_{i=1}^{d} S^{3}\right) \vee S^{5}$. Arguing as in Proposition 2.1 and using the fact from [16] that $\operatorname{Map}^{*}(X, Y)_{(1 / 2)} \simeq \operatorname{Map}^{*}\left(X_{(1 / 2)}, Y_{(1 / 2)}\right)$, we have the following. 
Proposition 2.2 If $M$ is a simply connected 4-manifold with $d$ two-cells which is not Spin, then there is a $\mathbb{Z}_{(1 / 2)}$-local decomposition

$$
\mathcal{G}_{k}(M, G) \simeq\left(\prod_{i=1}^{d} \Omega^{2} G\right) \times \mathcal{G}_{k} G .
$$

Next, recall that a compact, orientable Riemann surface $\Sigma_{g}$ of genus $g$ has a CWstructure with one $0-$ cell, $2 g$ 1-cells, and one 2-cell. So there is a homotopy cofibration sequence

$$
S^{1} \stackrel{f}{\longrightarrow} \bigvee_{i=1}^{2 g} S^{1} \stackrel{i}{\longrightarrow} \Sigma_{g} \stackrel{q}{\longrightarrow} S^{2}
$$

where $f$ attaches the top cell, $i$ is the inclusion, and $q$ pinches onto the top cell. It is well known that $f$ is the sum of Whitehead products $\bigoplus_{i=1}^{g}\left\langle a_{2 i-1}, a_{2 i}\right\rangle$ where $a_{j}$ is the inclusion of the $j$-th summand into the wedge $\bigvee_{i=1}^{2 g} S^{1}$. In particular, $\Sigma f$ is null homotopic and so $\Sigma \Sigma_{g} \simeq\left(\bigvee_{i=1}^{2 g} S^{2}\right) \vee S^{3}$. Let $\mathcal{G}_{k}\left(\Sigma_{g}, U(n)\right)=\Omega \operatorname{Map}_{k}\left(\Sigma_{g}, B U(n)\right)$ and $\mathcal{G}_{k} U(n)=\Omega \operatorname{Map}_{k}\left(S^{2}, B U(n)\right)$. Let $\mathbb{Z}^{2 g}$ be the product of $2 g$ copies of $\mathbb{Z}$.

Proposition 2.3 If $\Sigma_{g}$ is a compact, orientable Riemann surface of genus $g$ then there is an integral homotopy decomposition

$$
\mathcal{G}_{k, g} \simeq \mathbb{Z}^{2 g} \times\left(\prod_{i=1}^{2 g} \Omega \mathrm{SU}(n)\right) \times \mathcal{G}_{k} U(n) .
$$

Proof Argue as in Proposition 2.1, replacing the diagram in (2) with the analogous diagram induced by the pinch map $q: \Sigma_{g} \rightarrow S^{2}$. Also, since $U(n) \simeq S^{1} \times \mathrm{SU}(n)$, we have $\Omega U(n) \simeq \mathbb{Z} \times \Omega \mathrm{SU}(n)$, and so the decomposition $\mathcal{G}_{k}\left(\Sigma_{g}, U(n)\right) \simeq \mathcal{G}_{k} U(n) \times$ $\prod_{i=1}^{2 g} \Omega U(n)$ can be refined to the asserted one.

\section{Decompositions of Lie groups}

This section adds to what was said in the Introduction about decompositions of Lie groups. Recall that all spaces and maps have been localized at an odd prime $p$ and homology is taken with mod $-p$ coefficients. The Lie group $G$ is simple, simply connected, and compact, and its integral homology has no $p$-torsion. Of all the groups $\operatorname{Spin}(n), \operatorname{SU}(n), \operatorname{Sp}(n), G_{2}, F_{4}, E_{6}, E_{7}$, and $E_{8}$ and all the odd primes, the torsion free assumption eliminates only $F_{4}, E_{6}, E_{7}$ and $E_{8}$ at the prime 3, and $E_{8}$ at the prime 5. In the torsion free cases there is an isomorphism $H_{*}(G) \cong$ $\Lambda\left(x_{2 n_{1}+1}, \ldots, x_{2 n_{l}+1}\right)$, where $\left\{2 n_{1}-1, \ldots, 2 n_{l}-1\right\}$ is the type of $G$. There is a further isomorphism $H_{*}(G) \cong \bigotimes_{i=1}^{p-1} \Lambda\left(V_{i}\right)$ where $V_{i}$ consists of those generators 
in $\left\{x_{2 n_{1}+1}, \ldots, x_{2 n_{l}+1}\right\}$ whose degrees are of the form $2 i+j q+1$ for some $j \geq 0$. Based on work in $[15 ; 24 ; 23]$, it was shown in [28] that for each such $G$ there exists a space $A$ and a map $\imath: A \rightarrow G$ such that $H_{*}(G) \cong \Lambda\left(\tilde{H}_{*}(A)\right)$ and $l_{*}$ includes the generating set into the exterior algebra. For example, when $G=\operatorname{SU}(n)$ we have $A=\Sigma \mathbb{C} P^{n-1}$ and when $G=\operatorname{Sp}(n)$ we have $A$ equal to the space $Q_{n}$ constructed by James [17].

Theorem 3.1 states some properties of the decomposition in [28] of the groups $G$ in (1). For our purposes, the advantage of this decomposition over that in [23] is the existence of the factorization property in part (d). Note that the bounds on $n$ for the matrix groups in (1) depend on $(p-1)(p-2)$, whereas the statements in [28] had a bound on $n$ depending on $(p-1)(p-3)$. This was because the focus there was on a certain universal property satisfied by the factors, which we do not need here. To distill what is needed and why the stated bounds hold, we outline the proof. In what follows, we will refer to $S^{2 m+1}$ as an $H$-space. To specify the $H$-structure, recall that there is a homotopy fibration

$$
\Omega S^{4 m+1} \stackrel{\Omega w}{\longrightarrow} \Omega S^{2 m+2} \stackrel{\bar{r}}{\longrightarrow} S^{2 m+1}
$$

where $w$ is the Whitehead product of the identity map on $S^{2 m+2}$ with itself. The $H$-structure on $S^{2 m+1}$ we need is that induced from the fact that the suspension $E: S^{2 m+1} \rightarrow \Omega S^{2 m+2}$ is a left homotopy inverse of $\bar{r}$.

Theorem 3.1 Suppose that $G$ is one of the groups in (1). Then there are maps $t: \Sigma G \rightarrow \Sigma A$ and $r: \Omega \Sigma A \rightarrow \prod_{i=1}^{p-1} B_{i}$ such that

(a) $t$ is a left homotopy inverse of $\Sigma l: \Sigma A \rightarrow \Sigma G$;

(b) $B_{i}$ is a spherically resolved $H$-space, $H_{*}\left(B_{i}\right) \cong \Lambda\left(V_{i}\right)$, and if the highest dimensional element of $V$ has degree $2 m_{i}+1$, then there is an $H-m a p B_{i} \longrightarrow$ $S^{2 m_{i}+1}$;

(c) the composite

$$
e: G \stackrel{E}{\longrightarrow} \Omega \Sigma G \stackrel{\Omega t}{\longrightarrow} \Omega \Sigma A \stackrel{r}{\longrightarrow} \prod_{i=1}^{p-1} B_{i}
$$

is a homotopy equivalence;

(d) the map $r$ has the property that any $H-\operatorname{map} f: \Omega \Sigma A \rightarrow Z$ into a homotopy associative, homotopy commutative $H$-space $Z$ factors as

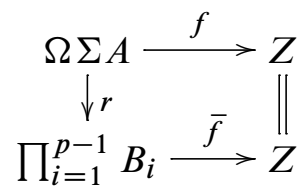

where $\bar{f}$ is an $H$-map. 
Proof The statements for the exceptional groups and part (a) are directly from [28]. For the matrix groups, by [24], there is a homotopy equivalence $A \simeq \bigvee_{i=1}^{p-1} A_{i}$ where $\widetilde{H}_{*}\left(A_{i}\right) \cong V_{i}$. The bound on $n$ in the statement of the theorem implies that each $A_{i}$ has at most $p-2$ cells, all of which are in odd dimensions. By [4], these conditions on the cells of $A_{i}$ imply that there is a spherically resolved $H$-space $B_{i}$ such that $H_{*}\left(B_{i}\right) \cong \Lambda\left(\tilde{H}_{*}\left(A_{i}\right)\right)$, and a homotopy fibration

$$
\Omega R_{i} \stackrel{\Omega \lambda_{i}}{\longrightarrow} \Omega \Sigma A_{i} \stackrel{r_{i}}{\longrightarrow} B_{i}
$$

where $r_{i}$ has a right homotopy inverse and $\lambda_{i}$ is defined via Whitehead products. Since the construction in [4] is functorial, the pinch map $A_{i} \longrightarrow S^{2 m_{i}+1}$ to the top cell induces a map of fibrations from $(*)$ to

$$
\Omega S^{4 m_{i}+1} \stackrel{\Omega w}{\longrightarrow} \Omega S^{2 m_{i}+2} \stackrel{\bar{r}}{\longrightarrow} S^{2 m_{i}+1},
$$

which implies that the induced map $B_{i} \longrightarrow S^{2 m_{i}+1}$ is an $H$-map. This proves part (b). The map $r$ is defined as the product of the maps $r_{i}$ for $1 \leq i \leq p-1$. Arguing as in [28, 1.1] shows that the composite $e$ is an isomorphism in homology and so is a homotopy equivalence, proving part (c). By the Hilton-Milnor Theorem, $\Omega \Sigma A \simeq \Omega\left(\bigvee_{i=1}^{p-1} \Sigma A_{i}\right)$ decomposes as $\prod_{i=1}^{p-1} \Omega \Sigma A_{i} \times P$, where $P$ is an infinite product of loop spaces, each factor of which maps into $\Omega \Sigma A$ by a looped Whitehead product. Putting things together, there is a homotopy fibration

$$
P \times \prod_{i=1}^{p-1} \Omega \Sigma R_{i} \stackrel{\lambda}{\longrightarrow} \Omega \Sigma A \stackrel{r}{\longrightarrow} \prod_{i=1}^{p-1} B_{i}
$$

where $\lambda$ is a product of looped Whitehead products. Further, the existence of a right homotopy inverse for each $r_{i}$ implies that $r$ has a right homotopy inverse. The factorization property in part (d) now follows as in [27, 5.3].

A more precise account of the factors of $\mathrm{SU}(n)$ was given in the Introduction. For $\operatorname{Sp}(n)$, the homotopy equivalence

$$
\mathrm{Sp}(n) \simeq \prod_{i=1}^{p-1} B_{i}
$$

simplifies as $H_{*}\left(B_{i}\right) \cong 0$ if $i$ is even, and so $B_{i}$ is contractible. If $i$ is odd, $H_{*}\left(B_{i}\right) \cong$ $\Lambda\left(x_{2 i+1}, x_{2 i+q+1}, \ldots, x_{2 i+\left(k_{n}^{\prime}-1\right) q+1}\right)$ for $k_{n}^{\prime}=\lfloor(2 n-i+1) /(p-1)\rfloor+1$. The 
decomposition of $\operatorname{Spin}(n)$ follows from that of $\operatorname{Sp}(n)$ and Harris' [15] decompositions

$$
\begin{aligned}
\operatorname{Spin}(2 n+1) & \simeq \operatorname{Sp}(n) \\
\operatorname{Spin}(2 n) & \simeq S^{2 n-1} \times \operatorname{Spin}(2 n-1) .
\end{aligned}
$$

Also, since $U(n) \simeq S^{1} \times \mathrm{SU}(n)$, the decomposition of $\mathrm{SU}(n)$ in Theorem 3.1 implies that for $n \leq(p-1)(p-2)+1$ there is a homotopy decomposition

$$
U(n) \simeq S^{1} \times \prod_{i=1}^{p-1} B_{i} .
$$

Note also in this case that there is a corresponding space $A=S^{1} \vee \Sigma \mathbb{C} P^{n-1}$ which satisfies $H_{*}(U(n)) \cong \Lambda\left(\widetilde{H}_{*}(A)\right)$.

One more general result is needed for what follows. This concerns being able to determine when two $H$-maps are homotopic. Let $J$ be the composite

$$
J: A \stackrel{\iota}{\longrightarrow} G \stackrel{e}{\longrightarrow} \prod_{i=1}^{p-1} B_{i} .
$$

By Theorem 3.1 (c), the map $r: \Omega \Sigma A \rightarrow \prod_{i=1}^{p-1} B_{i}$ has a right homotopy inverse. So by [12] we have the following.

Lemma 3.2 Suppose $Y$ is an $H$-space and $f, g: \prod_{i=1}^{p-1} B_{i} \longrightarrow Y$ are $H$-maps with the property that $f \circ \mathrm{J} \simeq g \circ \mathrm{J}$. Then $f \simeq g$.

\section{A reformulation of $\partial_{k}$}

In this section we prove Theorem 1.1. We first require two preliminary results. The first describes the adjoint of $\partial_{k}$ and was proved in [21]. Let $\iota: S^{3} \rightarrow G$ be the inclusion of the bottom cell. For $k \in \mathbb{Z}$, let $k: G \rightarrow G$ be the $k$-th power map. Write the composite $k \circ \iota$ as $k \iota$.

Lemma 4.1 The adjoint of the map $\partial_{k}: G \rightarrow \Omega_{0}^{3} G$ is homotopic to the Samelson product $\langle k \iota, 1\rangle: S^{3} \wedge G \rightarrow G$.

The second preliminary result concerns a decomposition of $\Sigma G$. By Theorem 3.1 the map $\Sigma l: \Sigma A \rightarrow \Sigma G$ has a right homotopy inverse, so $\Sigma G \simeq \Sigma A \vee C$ for some space $C$. In [13] the complementary factor $C$ is decomposed very finely. One consequence 
is given in Lemma 4.2. Let ev: $\Sigma G \simeq \Sigma \Omega B G \rightarrow B G$ be the evaluation map. Recall that there is a homotopy fibration

$$
\Sigma G \wedge G \stackrel{\mu^{*}}{\longrightarrow} \Sigma G \stackrel{\text { ev }}{\longrightarrow} B G
$$

where $\mu^{*}$ is the canonical Hopf construction.

Lemma 4.2 Let $G$ be one of the groups in (1) or let $G=U(n)$ and $A=S^{1} \vee \Sigma \mathbb{C} P^{n-1}$ when $n \leq(p-1)(p-2)+1$. Then there is a homotopy equivalence

$$
\Sigma A \vee C \stackrel{\Sigma \imath+s}{\longrightarrow} \Sigma G
$$

where $C$ is a retract of $\Sigma G \wedge G$ and $s$ factors through the Hopf construction $\mu^{*}$. $\square$

Let $\bar{l}$ be the composite

$$
\Sigma A \stackrel{\Sigma \imath}{\longrightarrow} \Sigma G \stackrel{\text { ev }}{\longrightarrow} B G .
$$

By Lemma 4.2, $\Sigma l$ has a left homotopy inverse $t: \Sigma G \rightarrow \Sigma A$. Further, since $s$ factors through the Hopf construction, it composes trivially with the evaluation map ev: $\Sigma G \rightarrow G$. Thus there is a homotopy commutative diagram

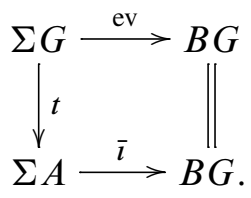

Proof of Theorem 1.1 By (3), for $k \in \mathbb{Z}$, the Whitehead product [kı, ev]: $\Sigma S^{3} \wedge G \rightarrow$ $B G$ factors through the Whitehead product $[k \iota, \bar{l}]: \Sigma S^{3} \wedge A \rightarrow B G$ and there is a homotopy commutative diagram

$$
\begin{aligned}
& \Sigma S^{3} \wedge G \stackrel{[k \iota, \mathrm{ev}]}{\longrightarrow} B G \\
& \begin{array}{l}
\vee^{\Sigma^{3} t} \\
\Sigma S^{3} \wedge A \stackrel{[k \iota, \bar{l}]}{\longrightarrow} B G .
\end{array}
\end{aligned}
$$

Let $s$ be the composite $s: G \stackrel{E}{\longrightarrow} \Omega \Sigma G \stackrel{\Omega t}{\longrightarrow} \Omega \Sigma A$. Consider the diagram

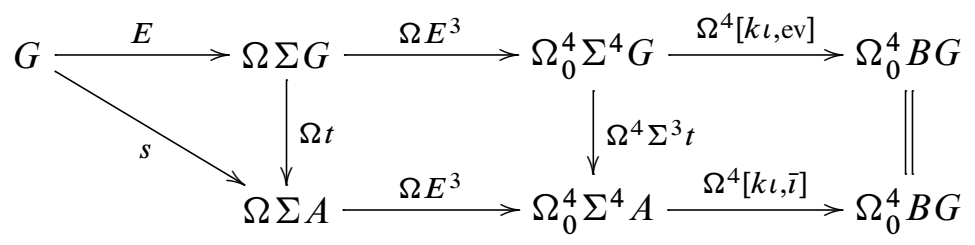


where $E^{3}$ is the triple suspension. The left triangle homotopy commutes by the definition of $s$, the middle square homotopy commutes by the naturality of $E^{3}$, and the right square homotopy commutes as it is the 4-fold loops of (4). Observe that the top row is homotopic to the 4 -fold right adjoint of $[k \iota, \mathrm{ev}]$, while $[k \iota, \mathrm{ev}]$ is homotopic to the 4 -fold left adjoint of $\partial_{k}$. Thus the top row is homotopic to $\partial_{k}$. Simplifying, there is a homotopy commutative diagram

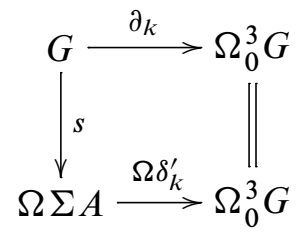

where $\delta_{k}^{\prime}=\Omega^{3}[k \iota, \bar{l}] \circ E^{3}$.

Since $\Omega \delta_{k}^{\prime}$ is an $H$-map, Theorem 3.1 implies that there is a homotopy commutative square

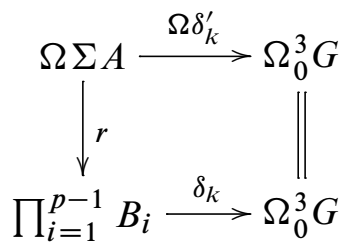

where $\delta_{k}$ is an $H$-map. Juxtaposing the previous two diagrams gives a homotopy $\partial_{k} \simeq \delta_{k} \circ r \circ s$. By the definitions of $s$ and $e, r \circ s$ is the homotopy equivalence $e$, and so the lemma has been proved.

Remark 4.3 An analogue of Lemma 4.2 holds for $U(n)$ and $A=S^{1} \vee \mathbb{C} P^{n-1}$ because there is a standard decomposition $U(n) \simeq S^{1} \times \operatorname{SU}(n)$. Replacing $\iota: S^{3} \rightarrow G$ with the inclusion $\bar{\imath}: S^{1} \rightarrow U(n)$ of the bottom cell, an argument similar to that in Theorem 1.1 can now be made to show that there is a homotopy commutative diagram

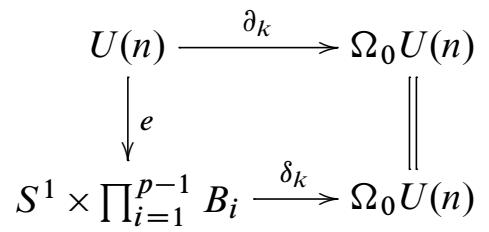

where $\delta_{k}$ is an $H$-map.

Theorem 1.1 plays a key role in the decompositions of $\mathcal{G}_{k} \mathrm{SU}(n), \mathcal{G}_{k} \mathrm{Sp}(n)$, and $\mathcal{G}_{k} U(n)$. For now, we give another application to show how it can be used in tandem with Lemma 3.2 to prove Proposition 1.2 and give some other decomposition results. 
Lemma 4.4 Let $G$ be one of the groups in (1). Suppose that the composite

$$
A \stackrel{\imath}{\longrightarrow} G \stackrel{\partial_{k}}{\longrightarrow} \Omega_{0}^{3} G
$$

is null homotopic. Then $\partial_{k}$ is null homotopic.

Proof By Theorem 1.1, it is equivalent to show that the map $\delta_{k}: \prod_{i=1}^{p-1} B_{i} \rightarrow \Omega_{0}^{3} G$ is null homotopic. The hypothesis $\partial_{k} \circ l \simeq *$ becomes equivalent to the composite

$$
A \stackrel{J}{\longrightarrow} \prod_{i=1}^{p-1} B_{i} \stackrel{\delta_{k}}{\longrightarrow} \Omega_{0}^{3} G
$$

being null homotopic. Compare $\delta_{k}$ and the trivial map. Both are $H$-maps, and by hypothesis, $\delta_{k} \circ \mathrm{J} \simeq * \simeq * \circ \mathrm{J}$. Thus Lemma 3.2 implies that $\delta_{k} \simeq *$.

Proof of Proposition 1.2 It is well known that $p>n_{l}$ implies that $G \simeq \prod_{i=1}^{l} S^{2 n_{i}-1}$. Noting that $2 n_{1}-1=3$ for all $G$, looping three times gives

$$
\Omega_{0}^{3} G \simeq \Omega^{3} S^{3}\langle 3\rangle \times \prod_{i=1}^{l} \Omega^{3} S^{2 n_{i}-1} .
$$

Since $\mathcal{G}_{k}$ is the homotopy fiber of the map $\partial_{k}: G \rightarrow \Omega_{0}^{3} G$, the proposition will follow if we show that $\partial_{k}$ is null homotopic. The condition $p>n_{l}$ guarantees that $G$ is one of the groups listed in (1). So by Lemma 4.4 it suffices to show that $\partial_{k} \circ l$ is null homotopic.

Observe that the decomposition of $G$ as a product of spheres implies that the space $A$ is homotopy equivalent to $\bigvee_{i=1}^{l} S^{2 n_{i}-1}$. So the adjoint of $\delta_{k} \circ l$ is a map $\Sigma^{3} A \longrightarrow G$ which can be replaced, up to homotopy equivalence, by $\bigvee_{i=1}^{l} S^{2 n_{i}+2} \rightarrow \prod_{i=1}^{l} S^{2 n_{i}-1}$. To show this is null homotopic, it is equivalent to show that each of the composites

$$
\theta_{a, b}: S^{2 n_{a}+2} \hookrightarrow \bigvee_{i=1}^{l} S^{2 n_{i}+2} \longrightarrow \prod_{i=1}^{l} S^{2 n_{i}-1} \rightarrow S^{2 n_{b}-1}
$$

is null homotopic for $1 \leq a, b \leq l$. Now $\pi_{m}\left(S^{2 n_{b}-1}\right)$ is torsion if $m \neq 2 n_{b}-1$, and by [30], the $p$-torsion class of least dimension occurs when $m=2 n_{b}+2 p-4$. Thus if $2 n_{a}+2<2 n_{b}+2 p-3$ for all $1 \leq a, b \leq l$ then $\pi_{2 n_{a}+2}\left(S^{2 n_{b}-1}\right)=0$ and so each $\theta_{a, b}$ is null homotopic. But this inequality is implied by the hypothesis that $p>n_{l}+1$. 
Sometimes $\partial_{k}$ can be shown to be null homotopic when $p \leq n_{l}+1$ simply by checking that the right homotopy groups of spheres are zero. As an example, consider the exceptional Lie group $E_{8}$. It has type $\{3,15,23,27,35,39,47,59\}$ so $n_{l}=30$. Thus Proposition 1.2 applies for primes $p \geq 37$. For primes $p \leq 31$ we have the following.

Proposition 4.5 For any $k \in \mathbb{Z}$, the map $\partial_{k}: E_{8} \rightarrow \Omega_{0}^{3} E_{8}$ is null homotopic when $p \in\{17,23,29\}$. Consequently, each case gives a $p$-local homotopy equivalence

$$
\mathcal{G}_{k} E_{8} \simeq E_{8} \times \Omega_{0}^{4} E_{8} .
$$

Proof We consider the $p=17$ case, the others being similar. By Lemma 4.4 it suffices to show that $\partial_{k} \circ l$ is null homotopic. Localized at $17, E_{8}$ decomposes as a product $B(3,35) \times B(15,47) \times B(27,59) \times S^{23} \times S^{39}$, where each $B(2 m+1,2 m+33)$ is a three-cell $H$-space whose bottom two cells are connected by the stable class $\alpha_{1}$. Let $A(2 m+1,2 m+33)$ be the $(2 m+33)$-skeleton of $B(2 m+1,2 m+33)$. The space $A$ in this case is the wedge $A(3,35) \vee A(15,47) \vee A(27,59) \vee S^{23} \vee S^{39}$.

The adjoint of $\partial_{k} \circ l$ is a map $f: \Sigma^{3} A \rightarrow E_{8}$. The cells of $\Sigma^{3} A$ are in dimensions $D=\{6,18,26,30,38,42,50,62\}$. So to show that $f$ is null homotopic it suffices to show that $\pi_{t}\left(E_{8}\right)=0$ for each $t \in D$. Using the decomposition of $E_{8}$ and the fact that there are homotopy fibrations $S^{2 m+1} \longrightarrow B(2 m+1,2 m+33) \longrightarrow S^{2 m+33}$ for each $2 m+1 \in\{3,15,27\}$, we will have $\pi_{t}\left(E_{8}\right)=0$ provided $\pi_{t}\left(S^{t^{\prime}}\right)=0$ for each $t \in D$ and $t^{\prime} \in\{3,15,23,27,35,39,47,59\}$. Checking Toda's [30] calculations of the odd primary homotopy groups of spheres shows that this is the case. Thus $f$ is null homotopic and so $\partial_{k} \circ l$ is null homotopic.

It may be the case that the map $\partial_{k}: E_{8} \rightarrow \Omega_{0}^{3} E_{8}$ is null homotopic for other primes $p \leq 37$ by different means. Also, Lemma 4.1 implies that $\partial_{k} \simeq k \partial_{1}$, so it may be the case that for large enough values of $k$ the potential obstructions to a null homotopy for $\partial_{k}$ are annihilated by multiplication by $k$. Other examples where $\partial_{k}$ is null homotopic for $p \leq n_{l}+1$ are $F_{4}$ and $E_{6}$ at 11 , and $E_{7}$ at 17 and 19 .

\section{A factorization related to the adjoint of $\partial_{k}$}

Let $\partial_{k}^{a}: S^{3} \wedge G \rightarrow G$ be the adjoint of $\partial_{k}: G \rightarrow \Omega_{0}^{3} G$. By Lemma 4.1, $\partial_{k}^{a}$ is homotopic to the Samelson product $\langle k \iota, 1\rangle$, which by linearity is homotopic to $k\langle\iota, 1\rangle \simeq k \partial_{1}^{a}$. In this section we specialize to $G=\mathrm{SU}(n)$ and prove an odd primary factorization of the composite

$$
S^{3} \wedge \Sigma \mathbb{C} P^{n-1} \stackrel{1 \wedge l}{\longrightarrow} S^{3} \wedge \mathrm{SU}(n) \stackrel{\partial_{1}^{a}}{\longrightarrow} \mathrm{SU}(n) .
$$


Corresponding factorizations for $\partial_{k}^{a} \circ(1 \wedge \imath)$ will follow by multiplying by $k$. Similar integral factorizations are proved with respect to the composites

$$
\begin{gathered}
S^{3} \wedge Q_{n} \stackrel{1 \wedge l}{\longrightarrow} S^{3} \wedge \operatorname{Sp}(n) \stackrel{\partial_{1}^{a}}{\longrightarrow} \operatorname{Sp}(n) \\
S^{1} \wedge \Sigma \mathbb{C} P^{n-1} \stackrel{1 \wedge l}{\longrightarrow} S^{1} \wedge U(n) \stackrel{\partial_{1}^{a}}{\longrightarrow} U(n) .
\end{gathered}
$$

Consider the homotopy fibration sequence

$$
\Omega(\mathrm{SU} / \mathrm{SU}(n)) \stackrel{g}{\longrightarrow} \mathrm{SU}(n) \stackrel{f}{\longrightarrow} \mathrm{SU} \longrightarrow \mathrm{SU} / \mathrm{SU}(n),
$$

where $\mathrm{SU}$ is the infinite special unitary group and $f$ is the canonical inclusion. Since $f$ is a group homomorphism it takes Samelson products to Samelson products. So as SU is homotopy commutative, the composite $f \circ\langle\iota, 1\rangle$ is null homotopic. Thus there is a lift

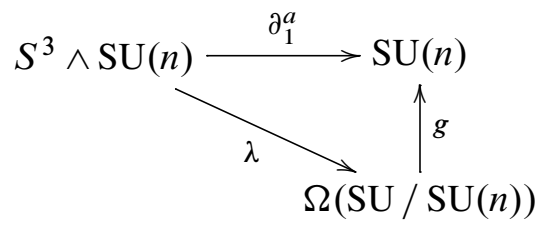

for some map $\lambda$. Consider the composite

$$
S^{3} \wedge \Sigma \mathbb{C} P^{n-1} \stackrel{1 \wedge l}{\longrightarrow} S^{3} \wedge \mathrm{SU}(n) \stackrel{\lambda}{\longrightarrow} \Omega(\mathrm{SU} / \mathrm{SU}(n)) .
$$

Since $\Omega(\mathrm{SU} / \mathrm{SU}(n))$ is $(2 n-1)$-connected, $\lambda \circ(1 \wedge l)$ collapses out the $(2 n-1)-$ skeleton of $S^{3} \wedge \Sigma \mathbb{C} P^{n-1}$. That is, if $M=\Sigma\left(\mathbb{C} P^{n-1} / \mathbb{C} P^{n-3}\right)$ is the suspension of the stunted projective space and $q: \Sigma \mathbb{C} P^{n-1} \rightarrow M$ is the quotient map, then there is a homotopy commutative diagram

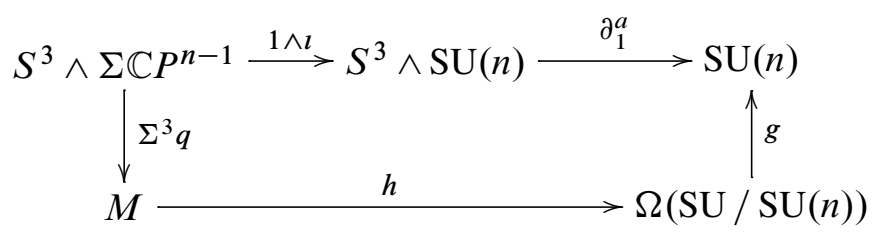

for some map $h$. Now we refine a bit. Observe that as a CW-complex, $M=$ $S^{2 n} \cup e^{2 n+2}$. Also, the $(2 n+2)-$ skeleton of $\Omega(\mathrm{SU} / \mathrm{SU}(n))$ is $S^{2 n} \cup e^{2 n+2}$. Localized at an odd prime both spaces are homotopy equivalent to $S^{2 n} \vee S^{2 n+2}$, and the map $h$ becomes the composite

$$
S^{2 n} \vee S^{2 n+2} \stackrel{a \vee b}{\longrightarrow} S^{2 n} \vee S^{2 n+2} \stackrel{\ell^{\prime}}{\longrightarrow} \Omega(\mathrm{SU} / \mathrm{SU}(n)),
$$


where $a$ and $b$ are some degree maps, and $\ell^{\prime}$ is the inclusion of the $(2 n+2)-$ skeleton. In Lemma 5.1 we show that both $a$ and $b$ equal \pm 1 .

In this section and the next, we need to consider $\pi_{2 n+2}(\mathrm{SU}(n))$, which is isomorphic integrally to $\mathbb{Z} / 12 \mathbb{Z}$ if $n=2$, to $\mathbb{Z} /(n+1) ! / 2 \mathbb{Z}$ if $n$ is odd, and to $\mathbb{Z} /(n+1)$ ! $\mathbb{Z} \oplus \mathbb{Z} / 2 \mathbb{Z}$ if $n \geq 4$ is even. For an odd prime $p$ the $p$-component in each case is the same as that of $\mathbb{Z} /(n+1) ! \mathbb{Z}$, so for brevity we will abuse notation by writing $\pi_{2 n+2}(\mathrm{SU}(n)) \cong$ $\mathbb{Z} /(n+1) ! \mathbb{Z}$.

Lemma 5.1 Let $n \geq 2$. After localizing at an odd prime, there is a homotopy commutative diagram

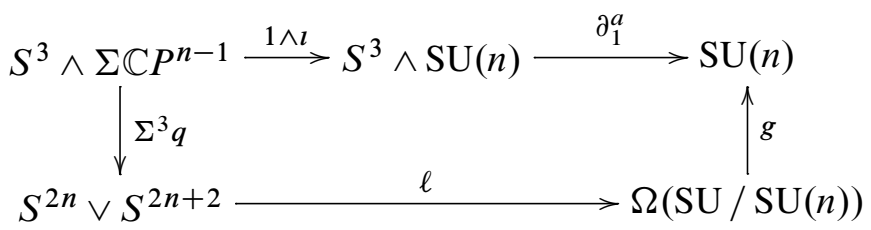

where $\ell$ is, up to signs, the inclusion of the bottom two cells.

Proof We begin with $b$. Let $q^{\prime}: M \rightarrow S^{2 n+2}$ be the pinch map onto the top cell. By [2], there is a characteristic map $S^{2 n-1} \longrightarrow \mathrm{SU}(n)$ with the property that the composite $S^{2 n-1} \longrightarrow \mathrm{SU}(n) \longrightarrow S^{2 n-1}$ is of degree $(n-1)$ !. By [29], the canonical map $\Sigma \mathbb{C} P^{n-1} \longrightarrow \mathrm{SU}(n)$ induces an isomorphism on the free part of $\pi_{*}(\mathrm{SU}(n))$. Thus there is a map $c: S^{2 n-1} \rightarrow \Sigma \mathbb{C} P^{n-1}$ with the property that the composite

$$
S^{2 n-1} \stackrel{c}{\longrightarrow} \Sigma \mathbb{C} P^{n-1} \stackrel{q}{\longrightarrow} M \stackrel{q^{\prime}}{\longrightarrow} S^{2 n-1}
$$

is of degree $(n-1)$ !. Consider the diagram

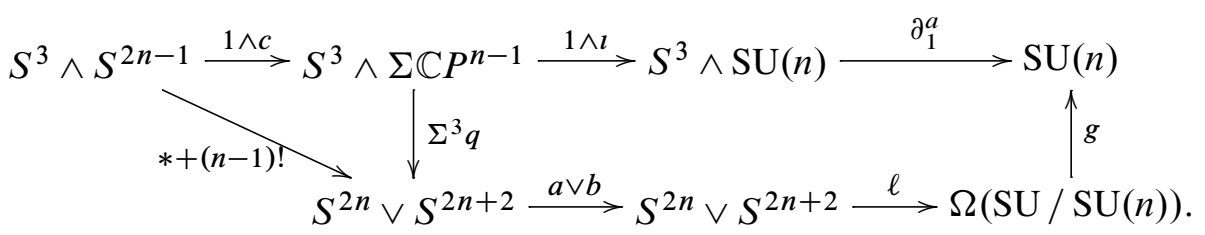

The left triangle homotopy commutes since $\pi_{2 n+2}\left(S^{2 n}\right)=0$ at odd primes. The right rectangle homotopy commutes by the refinement of (5). The composition $\partial_{1}^{a} \circ$ $(1 \wedge l) \circ(1 \wedge c)$ along the top row is the Samelson product $[l, c]$, which Bott [3] showed to have order $n(n+1)$. On the other hand, the restriction of $g \circ \ell$ to $S^{2 n+2}$ represents the generator of $\pi_{2 n+2}(\mathrm{SU}(n))=\mathbb{Z} /(n+1) ! \mathbb{Z}$. So the composite $g \circ \ell \circ$ $(a \vee b) \circ(*+(n-1) !)$ in the lower direction of the diagram has order $n(n+1) / b$. The commutativity of the diagram therefore implies that we map put $b= \pm 1$. 
For $a$, replace $1 \wedge c$ in (6) by the composite

$$
S^{3} \wedge S^{2 n-3} \stackrel{1 \wedge c^{\prime}}{\longrightarrow} S^{3} \wedge \Sigma \mathbb{C} P^{n-2} \stackrel{1 \wedge t}{\longrightarrow} S^{3} \wedge \Sigma \mathbb{C} P^{n-1}
$$

where $c^{\prime}$ is the characteristic map and $t$ is the inclusion. Now argue as before, using the facts that $\left\langle\iota, c^{\prime}\right\rangle$ has order $(n-1) n$ and $g \circ \ell$ restricted to $S^{2 n}$ represents the generator of $\pi_{2 n}(\mathrm{SU}(n))=\mathbb{Z} / n ! \mathbb{Z}$, to show that we may put $a= \pm 1$. Taking $\ell=\ell^{\prime} \circ(a \vee b)$ completes the proof.

The symplectic case is similar via the homotopy fibration sequence

$$
\Omega(\mathrm{Sp} / \mathrm{Sp}(n)) \stackrel{g}{\longrightarrow} \mathrm{Sp}(n) \longrightarrow \mathrm{Sp} \longrightarrow \mathrm{Sp} / \mathrm{Sp}(n) .
$$

As before, the Samelson product

$$
S^{3} \wedge \mathrm{Sp}(n) \stackrel{\partial_{1}^{a}}{\longrightarrow} \mathrm{Sp}(n)
$$

lifts through $g$ to a map $\lambda: S^{3} \wedge \mathrm{Sp}(n) \rightarrow \Omega(\operatorname{Sp} / \mathrm{Sp}(n))$. The composite

$$
S^{3} \wedge Q_{n} \stackrel{1 \wedge l}{\longrightarrow} S^{3} \wedge \operatorname{Sp}(n) \stackrel{\lambda}{\longrightarrow} \Omega(\operatorname{Sp} / \mathrm{Sp}(n))
$$

collapses out the $(4 n+1)$-skeleton since $\Omega(\operatorname{Sp} / \operatorname{Sp}(n))$ is $(4 n+1)$-connected. Thus $\lambda \circ(1 \wedge l)$ factors through the pinch map $\Sigma^{3} q: S^{3} \wedge Q_{n} \rightarrow S^{4 n+2}$ to the top cell, and so there is a homotopy commutative diagram

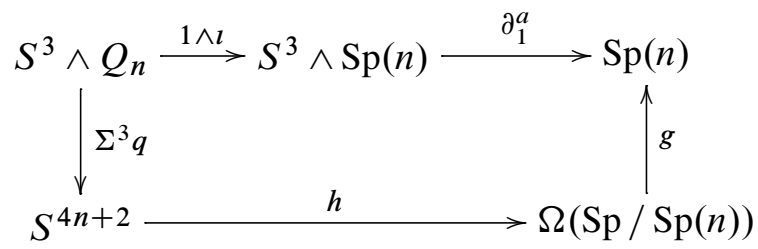

for some map $h$. For connectivity reasons, $h$ is homotopic to a composite

$$
S^{4 n+2} \stackrel{b}{\longrightarrow} S^{4 n+2} \stackrel{\ell^{\prime}}{\longrightarrow} \Omega(\operatorname{Sp} / \mathrm{Sp}(n))
$$

where $b$ is some degree map and $\ell^{\prime}$ is the inclusion of the bottom cell.

Lemma 5.2 Let $n \geq 1$. Integrally, there is a homotopy commutative diagram

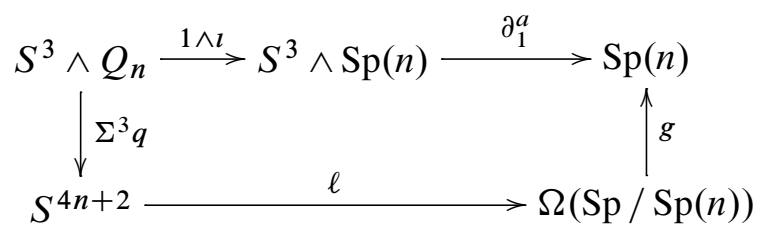


where $\ell$ is, up to sign, the inclusion of the bottom cell.

Proof We argue as in Lemma 5.1. By [2; 29], there is a characteristic map $c: S^{4 n-1} \rightarrow$ $Q_{n}$ which has the property that $q \circ c$ has degree $(2 n-1)$ ! if $n$ is odd and $2(2 n-1)$ ! if $n$ is even. There is a homotopy commutative diagram

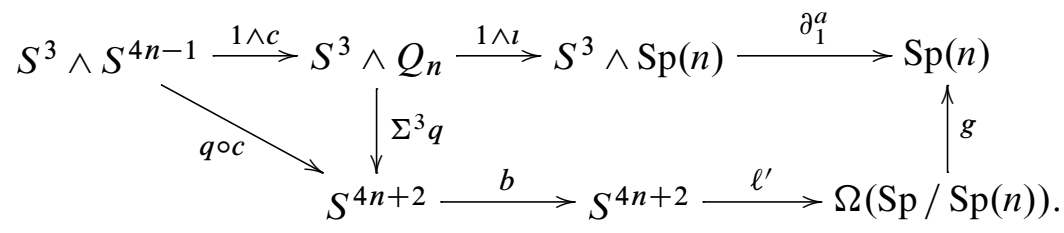

The composition $\partial_{1}^{a} \circ(1 \wedge l) \circ(1 \wedge c)$ along the top row is the Samelson product $\langle\iota, c\rangle$, which Bott [3] showed to have order a multiple of $4 n(2 n+1)$ if $n$ is odd and $n(2 n+1)$ if $n$ is even. On the other hand, the composite $g \circ \ell^{\prime}$ represents the generator of $\pi_{4 n+2}(\operatorname{Sp}(n))$, which equals $\mathbb{Z} / 2(2 n+1)$ ! $\mathbb{Z}$ if $n$ is odd and $\mathbb{Z} /(2 n+1)$ ! $\mathbb{Z}$ if $n$ is even. So the composite $g \circ \ell^{\prime} \circ b \circ(n-1)$ ! in the lower direction of the diagram has order $4 n(2 n+1) / b$ if $n$ is odd and $n(2 n+1) / b$ if $n$ is even. The commutativity of the diagram therefore implies that we may put $b= \pm 1$ for either $n$ odd or even. Taking $\ell=\ell^{\prime} \circ b$ completes the proof.

The case of $U(n)$-bundles over $S^{2}$ is analogous to the symplectic case. Consider the homotopy fibration sequence

$$
\Omega(U / U(n) \stackrel{g}{\longrightarrow} U(n) \longrightarrow U \longrightarrow U / U(n) .
$$

The Samelson product $\partial_{1}^{a}: S^{1} \wedge U(n) \rightarrow U(n)$ lifts through $g$ to a map $\lambda: S^{1} \wedge U(n) \rightarrow$ $\Omega(U / U(n))$. The restriction of $\lambda$ to $S^{1} \wedge \Sigma \mathbb{C} P^{n-1}$ collapses out the $(2 n-1)-$ skeleton since $\Omega(U / U(n))$ is $(2 n-1)$-connected. Thus $\lambda \circ(1 \wedge l)$ factors through the pinch map $\Sigma q: S^{1} \wedge \Sigma \mathbb{C} P^{n-1} \rightarrow S^{2 n}$ to the top cell, and so there is a homotopy commutative diagram

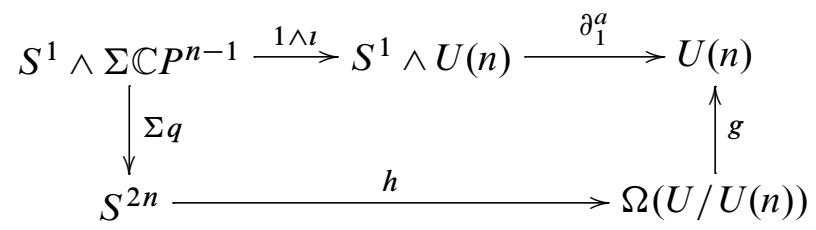

for some map $h$. For connectivity reasons, $h$ is homotopic to a composite

$$
S^{2 n} \stackrel{b}{\longrightarrow} S^{2 n} \stackrel{\ell^{\prime}}{\longrightarrow} \Omega(U / U(n))
$$

where $b$ is some degree map and $\ell^{\prime}$ is the inclusion of the bottom cell. 
Lemma 5.3 Let $n \geq 2$. Integrally, there is a homotopy commutative diagram

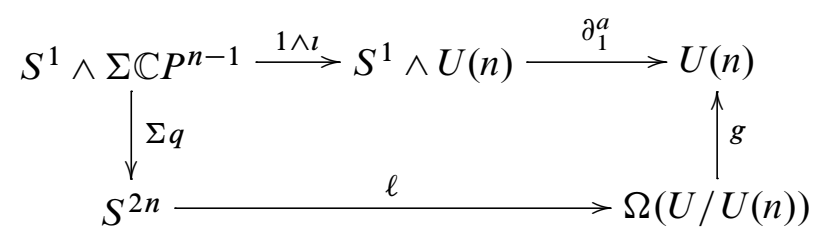

where $\ell$ is, up to sign, the inclusion of the bottom cell.

Proof We argue again as in Lemma 5.1. Consider the homotopy commutative diagram

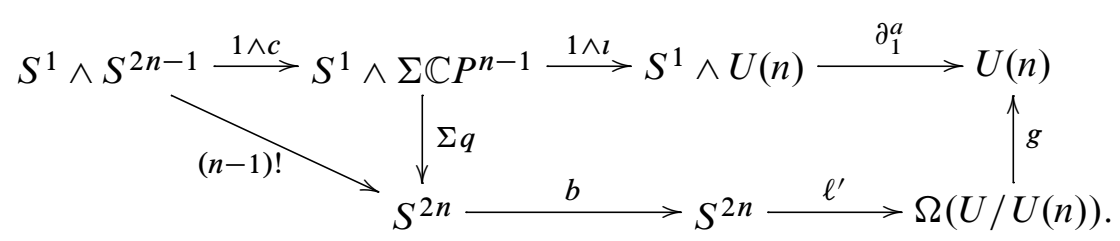

The composition $\partial_{1}^{a} \circ(1 \wedge l) \circ(1 \wedge c)$ along the top row is the Samelson product $\langle\iota, c\rangle$, which Bott [3] showed to have order $n$. On the other hand, $g \circ \ell^{\prime}$ represents the generator of $\pi_{2 n}(\mathrm{SU}(n))=\mathbb{Z} / n ! \mathbb{Z}$. So the composite $g \circ \ell^{\prime} \circ b \circ(n-1)$ ! in the lower direction of the diagram has order $n / b$. The commutativity of the diagram therefore implies that we may put $b= \pm 1$. Taking $\ell=\ell^{\prime} \circ b$ completes the proof.

\section{The decompositions}

In this section we prove the decomposition of $\mathcal{G}_{k} \mathrm{SU}(n)$ in Theorem 1.3, as well as analogous decompositions for $\mathcal{G}_{k} \operatorname{Sp}(n)$ and $\mathcal{G}_{k} U(n)$ in Theorems 6.2 and 6.3. Throughout we will assume spaces and maps have been localized at an odd prime.

First consider $\mathcal{G}_{k} \operatorname{SU}(n)$. We want to show that the $H$-map $\delta_{k}$ in Theorem 1.1 is homotopic to another $H$-map which is explicitly defined. Let $\gamma_{k}$ be the composite

$$
\begin{array}{r}
\gamma_{k}: \prod_{i=1}^{p-1} B_{i} \stackrel{\pi_{\mu, \nu}}{\longrightarrow} B_{\mu} \times B_{v} \longrightarrow S^{2 n-3} \times S^{2 n-1} \stackrel{E^{4} \times E^{4}}{\longrightarrow} \Omega^{4} S^{2 n+1} \times \Omega^{4} S^{2 n+3} \\
\stackrel{k \Omega^{3} \bar{s}_{n} \times k \Omega^{3} \bar{s}_{n+1}}{\longrightarrow} \Omega_{0}^{3} B_{u} \times \Omega_{0}^{3} B_{v} \stackrel{i_{u, v}}{\longrightarrow} \prod_{i=1}^{p-1} \Omega_{0}^{3} B_{i} \stackrel{\Omega^{3} e^{-1}}{\longrightarrow} \Omega_{0}^{3} \operatorname{SU}(n) .
\end{array}
$$

Here, $\pi_{\mu, \nu}$ is the projection onto the factors $B_{\mu}$ and $B_{v}$ carrying the degree $2 n-3$ and $2 n-1$ generators in homology; this is followed by the maps to the corresponding spheres; $E^{4}$ is the quadruple suspension; $i_{u, v}$ is the inclusion of the factors $B_{u}$ and $B_{v}$ 
which inherit from the equivalence $e$ the homotopy groups $\pi_{2 n}(\mathrm{SU}(n)) \cong \mathbb{Z} / n ! \mathbb{Z}$ and $\pi_{2 n+2}(\mathrm{SU}(n)) \cong \mathbb{Z} /(n+1) ! \mathbb{Z}$ respectively; and the maps $\bar{s}_{n}: \Omega S^{2 n+1} \rightarrow B_{u}$ and $\bar{s}_{n+1}: \Omega S^{2 n+3} \rightarrow B_{v}$ are extensions of representatives of those generators, the extensions existing because $B_{u}$ and $B_{v}$ are $H$-spaces. Numbering from left to right, the first, fourth, fifth and sixth maps in the composite defining $\gamma_{k}$ are clearly $H$-maps; the second map is an $H$-map by Theorem 3.1 (b); and in the third map each $E^{4}$ is an $H$-map with respect to the $H$-structure on an odd dimensional sphere defined just prior to Theorem 3.1. Thus $\gamma_{k}$ is an $H$-map as it is the composite of $H$-maps.

Lemma 6.1 There is a homotopy commutative diagram

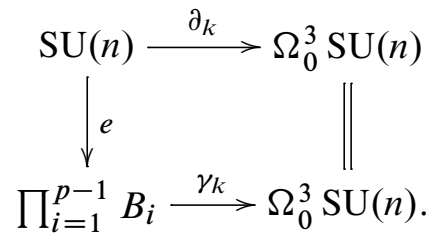

Proof The lemma follows immediately from Theorem 1.1 if we show that $\delta_{k}$ is homotopic to $\gamma_{k}$. Since both $\delta_{k}$ and $\gamma_{k}$ are $H$-maps, to show they are homotopic it suffices by Lemma 3.2 to show that $\delta_{k} \circ J$ is homotopic to $\gamma_{k} \circ J$, where $j$ is the composite

$$
\Sigma \mathbb{C} P^{n-1} \stackrel{\iota}{\longrightarrow} \mathrm{SU}(n) \stackrel{e}{\longrightarrow} \prod_{i=1}^{p-1} B_{i} .
$$

Recasting, Theorem 1.1 states that $\delta_{k} \circ J \simeq \partial_{k} \circ l$, so it is equivalent to show that $\gamma_{k} \circ \mathrm{J}$ is homotopic to $\partial_{k} \circ l$.

First consider $\gamma_{k} \circ J$. By connectivity, the composite

$$
\Sigma \mathbb{C} P^{n-1} \stackrel{J}{\longrightarrow} \prod_{i=1} B_{i} \stackrel{\pi_{\mu, v}}{\longrightarrow} B_{u} \times B_{v} \longrightarrow S^{2 n-3} \times S^{2 n-1}
$$

is homotopic to the composite

$$
\Sigma \mathbb{C} P^{n-1} \stackrel{q}{\longrightarrow} S^{2 n-3} \vee S^{2 n-1} \stackrel{w}{\longrightarrow} S^{2 n-3} \times S^{2 n-1}
$$

where $w$ is the inclusion of the wedge into the product. Thus $\gamma_{k} \circ \mathrm{J} \simeq \Omega^{3} e^{-1} \circ i_{u, v} \circ$ $\left(E^{4} \times E^{4}\right) \circ\left(k \Omega^{3} \bar{s}_{n} \times k \Omega^{3} \bar{s}_{n+1}\right) \circ w \circ q$.

Next consider $\partial_{k} \circ \imath$. Return to the diagram in Lemma 5.1. Let $s_{n}$ and $s_{n+1}$ be the restrictions of

$$
S^{2 n} \vee S^{2 n+2} \stackrel{\ell}{\longrightarrow} \Omega(\mathrm{SU} / \mathrm{SU}(n)) \stackrel{g}{\longrightarrow} \mathrm{SU}(n)
$$


to $S^{2 n}$ and $S^{2 n+2}$ respectively. So $s_{n}$ and $s_{n+1}$ represent the generators of the groups $\pi_{2 n}(\mathrm{SU}(n)) \cong \mathbb{Z} / n ! \mathbb{Z}$ and $\pi_{2 n+2}(\mathrm{SU}(n)) \cong \mathbb{Z} /(n+1) ! \mathbb{Z}$. Lemma 5.1 states that there is a homotopy $\partial_{1}^{a} \circ(1 \wedge l) \simeq\left(s_{n}+s_{n+1}\right) \circ \Sigma^{3} q$. Composing with the $k$-th power map on $\mathrm{SU}(n)$ gives $k \partial_{1}^{a} \circ(1 \wedge l) \simeq\left(k s_{n}+k s_{n+1}\right) \circ \Sigma^{3} q$. Since Lemma 4.1 implies that $\partial_{k}^{a} \simeq k \partial_{1}^{a}$ we obtain a homotopy commutative diagram

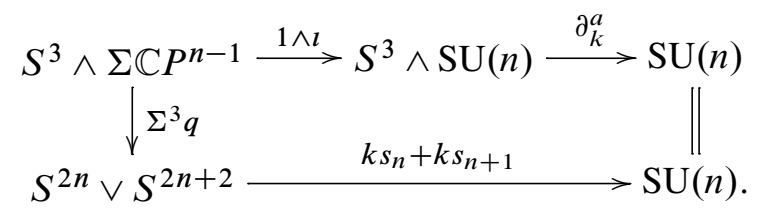

Now introduce the decomposition $e: \operatorname{SU}(n) \rightarrow \prod_{i=1}^{p-1} B_{i}$ in Theorem 3.1. Since $s_{n}$ and $s_{n+1}$ represent the generators of $\pi_{2 n}(\mathrm{SU}(n))$ and $\pi_{2 n+1}(\mathrm{SU}(n))$, the definition of the maps $\bar{s}_{n}$ and $\bar{s}_{n+1}$ implies that the composite

$$
S^{2 n} \vee S^{2 n+1} \stackrel{k s_{n}+k s_{n+1}}{\longrightarrow} \mathrm{SU}(n) \stackrel{e}{\longrightarrow} \prod_{i=1}^{p-1} B_{i}
$$

is homotopic to the composite

$$
\begin{aligned}
\epsilon: S^{2 n} \vee S^{2 n+1} \longrightarrow S^{2 n} \times S^{2 n+1} & \stackrel{E \times E}{\longrightarrow} \Omega S^{2 n+1} \times \Omega S^{2 n+3} \\
& \stackrel{k \bar{s}_{n} \times k \bar{s}_{n+1}}{\longrightarrow} B_{u} \times B_{v} \stackrel{i_{u, v}}{\longrightarrow} \prod_{i=1}^{p-1} B_{i} .
\end{aligned}
$$

Combining this homotopy with (9) gives $e \circ \partial_{k}^{a} \circ(1 \wedge l) \simeq \epsilon \circ \Sigma^{3} q$. Now take the 3 -fold right adjoint, and use the naturality of adjointing with the 3 -fold suspension to obtain a homotopy commutative diagram

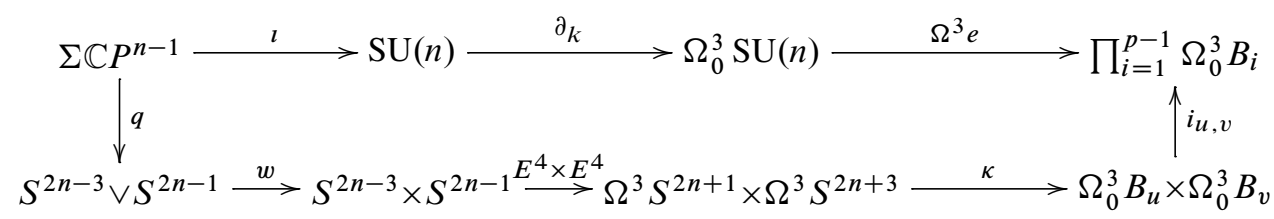

here $\kappa=k \Omega^{3} \bar{s}_{n} \times k \Omega^{3} \bar{s}_{n+1}$. Equivalently, $\partial_{k} \circ l \simeq \Omega^{3} e^{-1} \circ i_{u, v} \circ\left(E^{4} \times E^{4}\right) \circ$ $\left(k \Omega^{3} \bar{s}_{n} \times k \Omega^{3} \bar{s}_{n+1}\right) \circ w \circ q$. Hence $\partial_{k} \circ l \simeq \gamma_{k} \circ \mathrm{J}$, as required.

Now we are ready to decompose $\mathcal{G}_{k} \mathrm{SU}(n)$. Let $X_{k}$ and $Y_{k}$ respectively be the homotopy fibers of the composites

$$
\begin{aligned}
& B_{\mu} \longrightarrow S^{2 n-3} \stackrel{E^{4}}{\longrightarrow} \Omega^{4} S^{2 n+1} \stackrel{k \Omega^{3} \bar{s}_{n}}{\longrightarrow} \Omega_{0}^{3} B_{u} \\
& B_{v} \longrightarrow S^{2 n-1} \stackrel{E^{4}}{\longrightarrow} \Omega^{4} S^{2 n+3} \stackrel{k \Omega^{3} \bar{s}_{n+1}}{\longrightarrow} \Omega_{0}^{3} B_{v} .
\end{aligned}
$$


Proof of Theorem 1.3 The space $\mathcal{G}_{k} \mathrm{SU}(n)$ is the homotopy fiber of the map $\partial_{k}$. By Lemma 6.1, $\mathcal{G}_{k} \mathrm{SU}(n)$ is homotopy equivalent to the homotopy fiber of $\gamma_{k}$. Eliminating the equivalence $\Omega^{3} e^{-1}$ in the definition of $\gamma_{k}$ shows that $\mathcal{G}_{k} \mathrm{SU}(n)$ is homotopy equivalent to the homotopy fiber of the composite

$$
\begin{aligned}
\prod_{i=1}^{p-1} B_{i} \stackrel{\pi_{\mu, \nu}}{\longrightarrow} B_{u} \times B_{v} \longrightarrow S^{2 n-3} \times S^{2 n-1} \stackrel{E^{4} \times E^{4}}{\longrightarrow} \Omega^{4} S^{2 n+1} \times \Omega^{4} S^{2 n+3} \\
\stackrel{k \Omega^{3} \bar{s}_{n} \times k \Omega^{3} \bar{s}_{n+1}}{\longrightarrow} \Omega_{0}^{3} B_{u} \times \Omega_{0}^{3} B_{v} \stackrel{i_{u, v}}{\longrightarrow} \prod_{i=1}^{p-1} \Omega_{0}^{3} B_{i} .
\end{aligned}
$$

Since the initial arrow is a projection and the final arrow is an inclusion, the asserted decomposition of $\mathcal{G}_{k} \mathrm{SU}(n)$ follows immediately.

The case of $\mathcal{G}_{k} \operatorname{Sp}(n)$ is similar. The map $\gamma_{k}$ takes the form

$$
\begin{aligned}
\gamma_{k}: \prod_{i=1}^{p-1} B_{i} \stackrel{\pi_{\mu}}{\longrightarrow} B_{\mu} \longrightarrow S^{4 n-1} \stackrel{E^{4}}{\longrightarrow} \Omega^{4} S^{4 n+3} \\
\stackrel{k \Omega^{3} \bar{s}_{n}}{\longrightarrow} \Omega_{0}^{3} B_{u} \stackrel{i_{u}}{\longrightarrow} \prod_{i=1}^{p-1} \Omega_{0}^{3} B_{i} \stackrel{\Omega^{3} e^{-1}}{\longrightarrow} \Omega_{0}^{3} \operatorname{Sp}(n) .
\end{aligned}
$$

The diagram in Lemma 6.1 becomes

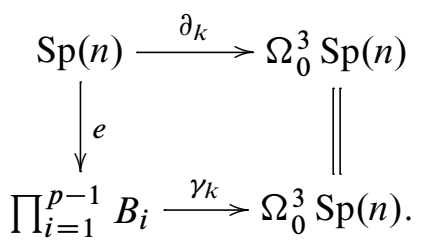

Let $X_{k}$ be the homotopy fiber of the composite

$$
B_{\mu} \longrightarrow S^{4 n-1} \stackrel{E^{4}}{\longrightarrow} \Omega^{4} S^{4 n+3} \stackrel{k \Omega^{3} s_{n}}{\longrightarrow} \Omega_{0}^{3} B_{u}
$$

Arguing as in Theorem 1.3 we obtain the following.

Theorem 6.2 There is a homotopy decomposition

$$
\mathcal{G}_{k} \operatorname{Sp}(n) \simeq X_{k} \times\left(\prod_{\substack{j=1 \\ j \neq \mu}}^{p-1} B_{j}\right) \times\left(\prod_{\substack{i=1 \\ i \neq \mu}}^{p-1} \Omega_{0}^{4} B_{i}\right) .
$$


In the case of $\mathcal{G}_{k} U(n)$ the map $\gamma_{k}$ takes the form

$$
\begin{aligned}
\gamma_{k}: S^{1} \times \prod_{i=1}^{p-1} B_{i} \stackrel{\pi_{\mu}}{\longrightarrow} B_{\mu} \longrightarrow S^{2 n-1} \stackrel{E^{2}}{\longrightarrow} \Omega^{2} S^{2 n+1} \\
\quad \stackrel{k \Omega \bar{s}_{n}}{\longrightarrow} \Omega B_{u} \stackrel{i_{u}}{\longrightarrow} \prod_{i=1}^{p-1} \Omega B_{i} \stackrel{\Omega e^{-1}}{\longrightarrow} \Omega_{0} U(n) .
\end{aligned}
$$

The diagram in Lemma 6.1 becomes

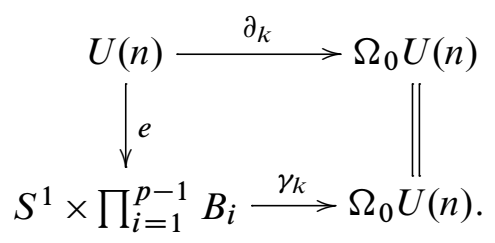

Let $X_{k}$ be the homotopy fiber of the composite

$$
B_{\mu} \longrightarrow S^{2 n-1} \stackrel{E^{2}}{\longrightarrow} \Omega^{2} S^{2 n+1} \stackrel{k \Omega s_{n}}{\longrightarrow} \Omega B_{u} .
$$

Arguing as in Theorem 1.3 we obtain the following.

Theorem 6.3 There is a homotopy decomposition

$$
\mathcal{G}_{k} U(n) \simeq S^{1} \times X_{k} \times\left(\prod_{\substack{j=1 \\ j \neq \mu}}^{p-1} B_{j}\right) \times\left(\prod_{\substack{i=1 \\ i \neq \mu}}^{p-1} \Omega^{2} B_{i}\right) .
$$

\section{Spin-gauge groups}

In this section we only assume that $p$ is odd, without any restriction on the dimension parameter $n$. In Section 3 we saw that there are $p$-local homotopy equivalences $\operatorname{Spin}(2 n+1) \simeq \operatorname{Sp}(n)$ and $\operatorname{Spin}(2 n) \simeq S^{2 n-1} \times \operatorname{Sp}(n-1)$. We will show that these equivalences determine $p$-local homotopy equivalences $\mathcal{G}_{k} \operatorname{Spin}(2 n+1) \simeq \mathcal{G}_{k} \operatorname{Sp}(n)$ and $\mathcal{G}_{k} \operatorname{Spin}(2 n) \simeq S^{2 n-1} \times \Omega^{4} S^{2 n+3} \times \mathcal{G}_{k} \operatorname{Sp}(n-1)$. Further decompositions then exist by Theorem 6.2.

Theorem 7.1 For $n \geq 1$ there is a $\mathbb{Z}_{(1 / 2)}$-local homotopy equivalence

$$
\mathcal{G}_{k} \operatorname{Spin}(2 n+1) \simeq \mathcal{G}_{k} \operatorname{Sp}(n)
$$

Proof Friedlander [10] showed that there is a $\mathbb{Z}_{(1 / 2)}$-local homotopy equivalence $f: B \operatorname{Spin}(2 n+1) \rightarrow B \operatorname{Sp}(n)$. This map does not exist integrally. Observe that the 
induced map $\pi_{4}(B \operatorname{Spin}(2 n+1)) \otimes \mathbb{Z}_{(1 / 2)} \longrightarrow \pi_{4}(B \operatorname{Sp}(n)) \otimes \mathbb{Z}_{(1 / 2)}$ is an isomorphism, implying that $f^{*}$ sends $k \in \pi_{4}(B \operatorname{Spin}(2 n+1))$ to $2^{j} k \in \pi_{4}(B \operatorname{Sp}(n))$ for some integer $j$, possibly negative. By [16], there is a homotopy equivalence

$$
\begin{aligned}
B \mathcal{G}_{k} \operatorname{Spin}(2 n+1)_{(1 / 2)} & =\operatorname{Map}_{k}\left(S^{4}, B \operatorname{Spin}(2 n+1)\right)_{(1 / 2)} \\
& \simeq \operatorname{Map}_{k}\left(S^{4}, B \operatorname{Spin}(2 n+1)_{(1 / 2)}\right)
\end{aligned}
$$

and similarly $B \mathcal{G}_{k} \operatorname{Sp}(n)_{(1 / 2)} \simeq \operatorname{Map}_{k}\left(S^{4}, B \operatorname{Sp}(n)_{(1 / 2)}\right)$. Thus $f$ induces a morphism between $\mathbb{Z}_{(1 / 2)}$-local evaluation fibrations $\operatorname{Map}_{k}\left(S^{4}, B \operatorname{Spin}(2 n+1)\right) \longrightarrow$ $B \operatorname{Spin}(2 n+1)$ and $\operatorname{Map}_{2^{j} k}\left(S^{4}, B \operatorname{Sp}(n)\right) \longrightarrow B \operatorname{Sp}(n)$ - we suppress the $\mathbb{Z}_{(1 / 2)}$ now - which induces a homotopy commutative diagram of fibration connecting maps

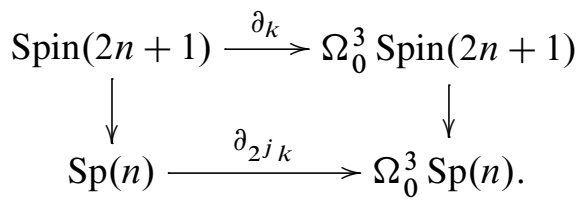

Both vertical maps in this diagram are $\mathbb{Z}_{(1 / 2)}$-local homotopy equivalences, and so induce a $\mathbb{Z}_{(1 / 2)}$-local homotopy equivalence $\mathcal{G}_{k} \operatorname{Spin}(2 n+1) \simeq \mathcal{G}_{2^{j} k} \operatorname{Sp}(n)$ between fibers.

To complete the proof, we will show that there is a $\mathbb{Z}_{(1 / 2)}$-local homotopy equivalence $\mathcal{G}_{2^{j} k} \operatorname{Sp}(n) \simeq \mathcal{G}_{k} \operatorname{Sp}(n)$. By Lemma 4.1 and the linearity of the Samelson product, for any integer $m$ there is a homotopy $\partial_{m} \simeq m \circ \partial_{1}$ where the map $m$ is the $m$-th power map on $\Omega_{0}^{3} \operatorname{Sp}(n)$. Now suppose that $j \geq 0$. Then $\partial_{2^{j} k} \simeq 2^{j} \circ \partial_{k}$, so there is a homotopy fibration diagram

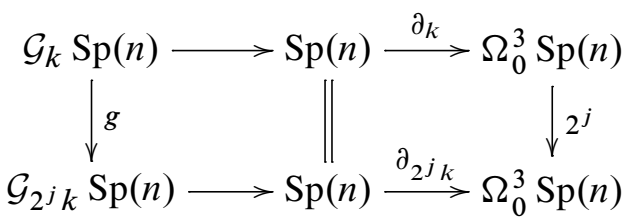

for some induced map $g$ of fibers. Localizing at $\mathbb{Z}_{(1 / 2)}$, the $2^{j}$-power map is a homotopy equivalence, so the five-lemma implies that $g$ is a $\mathbb{Z}_{(1 / 2)}$-local homotopy equivalence. The argument for $j<0$ is similar, using the fact that if $k=2^{-j} k^{\prime}$, then $\partial_{k} \simeq 2^{-j} \circ \partial_{k^{\prime}}$.

Next, consider the map $\partial_{k}: \operatorname{Spin}(2 n) \rightarrow \Omega_{0}^{3} \operatorname{Spin}(2 n)$, or equivalently

$$
\partial_{k}: S^{2 n-1} \times \operatorname{Spin}(2 n-1) \rightarrow \Omega^{3} S^{2 n-1} \times \Omega_{0}^{3} \operatorname{Spin}(2 n-1) .
$$

After some preliminary work, Lemma 7.3 reduces this to the case of $\partial_{k}: \operatorname{Sp}(n-1) \rightarrow$ $\Omega_{0}^{3} \operatorname{Sp}(n-1)$. 
Lemma 7.2 Let $n \geq 2$. Suppose $p \geq 5$ or $p=3$ and $k$ is a multiple of 3 . Then the inclusion $S^{2 n-1} \longrightarrow \operatorname{Spin}(2 n)$ lifts to a map

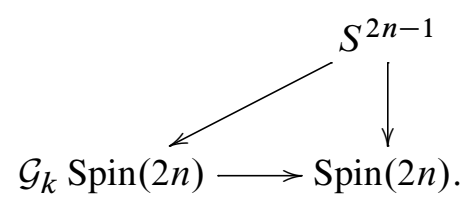

Proof We use the canonical fibration $\operatorname{Spin}(2 n-1) \longrightarrow \operatorname{Spin}(2 n) \longrightarrow S^{2 n-1}$, which splits at odd primes. Consider the composite

$$
f: S^{2 n-1} \hookrightarrow S^{2 n-1} \times \operatorname{Sp}(2 n-1) \stackrel{\simeq}{\longrightarrow} \operatorname{Spin}(2 n) \stackrel{\partial_{k}}{\longrightarrow} \Omega_{0}^{3} \operatorname{Spin}(2 n) .
$$

Adjointing gives a map $f^{a}: S^{2 n+2} \rightarrow \operatorname{Spin}(2 n)$. We claim that the composite

$$
S^{2 n+2} \stackrel{f^{a}}{\longrightarrow} \operatorname{Spin}(2 n) \longrightarrow S^{2 n-1}
$$

is null homotopic. This is the case if $p \geq 5$ because then $\pi_{2 n+2}\left(S^{2 n-1}\right)=0$. If $p=3$ then $\pi_{2 n+2}\left(S^{2 n-1}\right) \cong \mathbb{Z} / 3 \mathbb{Z}$, so there is a potential obstruction to a null homotopy for $f^{a}$. By [18] (see Remark 7.5), this is in fact a genuine obstruction. But if $k$ is a multiple of 3 then, as Lemma 4.1 implies that $\partial_{k}^{a} \simeq k \partial_{1}^{a}$, the factorization of $f^{a}$ through $\partial_{k}^{a}$ implies that $f^{a}$ is divisible by 3 . Thus $f^{a}$ annihilates $\pi_{2 n+2}\left(S^{2 n-1}\right)$ and so is null homotopic. In either case, the null homotopy for $f^{a}$ implies that it lifts through the inclusion $\operatorname{Spin}(2 n-1) \longrightarrow \operatorname{Spin}(2 n)$. By Lemma 4.1, the adjoint of $\partial_{k}$ is homotopic to a Samelson product and so composes trivially into $\operatorname{Spin}(\infty)$ as this space is homotopy commutative. Thus $f^{a}$, which factors through $\partial_{k}^{a}$, composes trivially into $\operatorname{Spin}(\infty)$. Therefore, the lift of $f^{a}$ through $\operatorname{Spin}(2 n-1)$ lifts further to a map $S^{2 n-1} \longrightarrow \Omega(\operatorname{Spin}(\infty) / \operatorname{Spin}(2 n-1))$. This map is null homotopic since $\Omega(\operatorname{Spin}(\infty) / \operatorname{Spin}(2 n-1))$ is $(4 n-6)$-connected. Thus $f^{a}$ is null homotopic, and so $f$ is null homotopic. The existence of the asserted lift to $\mathcal{G}_{k} \operatorname{Spin}(2 n)$ follows immediately.

Lemma 7.3 Let $n \geq 2$. Suppose $p \geq 5$ or $p=3$ and $k$ is a multiple of 3 . Then there is a homotopy commutative diagram

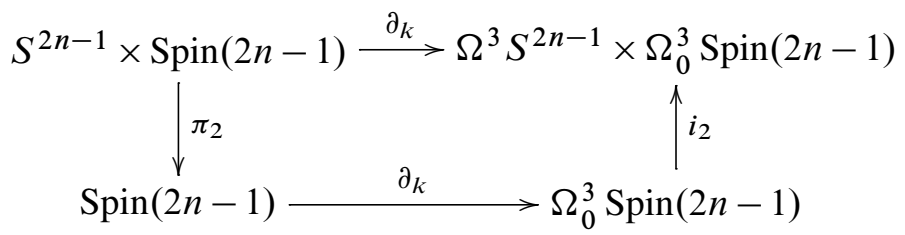

where $\pi_{2}$ is the projection and $i_{2}$ is the inclusion. 
Proof Since $\partial_{k}: \operatorname{Spin}(2 n) \rightarrow \Omega_{0}^{3} \operatorname{Spin}(2 n)$ is a fibration connecting map, there is a homotopy action $\theta: \Omega_{0}^{3} \operatorname{Spin}(2 n) \times \operatorname{Spin}(2 n) \rightarrow \Omega_{0}^{3} \operatorname{Spin}(2 n)$. Using the lift from Lemma 7.2, consider the diagram

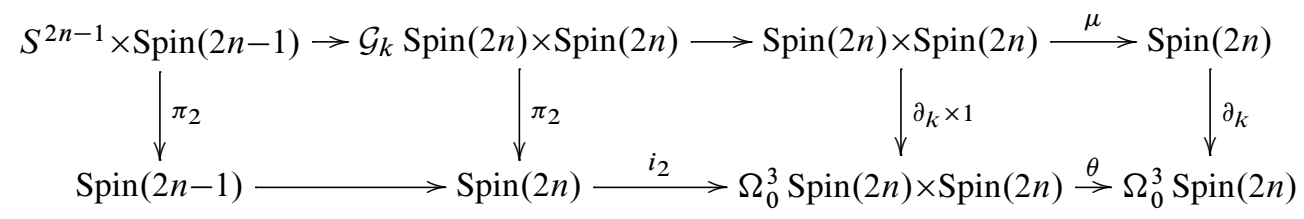

where $\mu$ is the loop multiplication. The left square homotopy commutes by the naturality of the projection, the middle square homotopy commutes since

$$
\mathcal{G}_{k} \operatorname{Spin}(2 n) \longrightarrow \operatorname{Spin}(2 n) \stackrel{\partial_{k}}{\longrightarrow} \Omega_{0}^{3} \operatorname{Spin}(2 n)
$$

is a homotopy fibration and so the composite is null homotopic. The right square homotopy commutes because it is a canonical property of the homotopy action $\theta$. Thus the diagram as a whole homotopy commutes. Now observe that the composite along the top row is a homotopy equivalence. The composite along the bottom row simplifies to

$$
a: \operatorname{Spin}(2 n-1) \longrightarrow \operatorname{Spin}(2 n) \stackrel{\partial_{k}}{\longrightarrow} \Omega_{0}^{3} \operatorname{Spin}(2 n) .
$$

Suppose we knew that $a$ is homotopic to the composite

$$
b: \operatorname{Spin}(2 n-1) \stackrel{\partial_{k}}{\longrightarrow} \Omega_{0}^{3} \operatorname{Spin}(2 n-1) \longrightarrow \Omega_{0}^{3} \operatorname{Spin}(2 n) .
$$

Then the outer rectangle of this diagram would be the diagram asserted by the lemma. To show that $a$ is homotopic to $b$, observe that the inclusion $\operatorname{Spin}(2 n-1) \longrightarrow \operatorname{Spin}(2 n)$ is a group homomorphism and so induces a map $B \operatorname{Spin}(2 n-1) \longrightarrow B \operatorname{Spin}(2 n)$. The naturality of the evaluation fibration $\operatorname{Map}_{k}(X, B G) \longrightarrow B G$ therefore implies that there is a homotopy commutative diagram of connecting maps

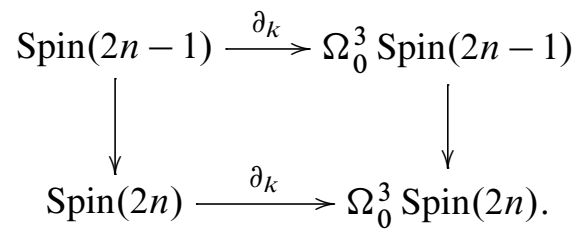

The upper direction around this square is the definition of $b$ and the lower direction is the definition of $a$, and so $a \simeq b$, as required.

The factorization in Lemma 7.3 immediately gives the following. 
Theorem 7.4 Let $n \geq 2$. Suppose $p \geq 5$ or $p=3$ and $k$ is a multiple of 3 . Then there is a $p$-local homotopy decomposition

$$
\mathcal{G}_{k} \operatorname{Spin}(2 n) \simeq S^{2 n-1} \times \Omega^{4} S^{2 n-1} \times \mathcal{G}_{k} \operatorname{Spin}(2 n-1) .
$$

Remark 7.5 After this paper appeared, the decomposition in Theorem 7.4 was also proved by Kishimoto and Kono [18] using different methods. In addition, they determine what happens when $p=3$ and $k$ is prime to 3: there is a homotopy decomposition $\mathcal{G}_{k} \operatorname{Spin}(2 n) \simeq X \times \mathcal{G}_{k} \operatorname{Spin}(2 n-1)$ where $X$ is known to not be homotopy equivalent to $S^{2 n-1} \times \Omega^{4} S^{2 n-1}$.

\section{References}

[1] M F Atiyah, R Bott, The Yang-Mills equations over Riemann surfaces, Philos. Trans. Roy. Soc. London Ser. A 308 (1983) 523-615 MR702806

[2] A Borel, F Hirzebruch, Characteristic classes and homogeneous spaces. I, Amer. J. Math. 80 (1958) 458-538 MR0102800

[3] R Bott, A note on the Samelson product in the classical groups, Comment. Math. Helv. 34 (1960) 249-256 MR0123330

[4] F R Cohen, J A Neisendorfer, A construction of $p$-local $H$-spaces, from: "Algebraic topology, Aarhus 1982 (Aarhus, 1982)", (I Madsen, B Oliver, editors), Lecture Notes in Math. 1051, Springer, Berlin (1984) 351-359 MR764588

[5] R L Cohen, R J Milgram, The homotopy type of gauge-theoretic moduli spaces, from: "Algebraic topology and its applications", (GE Carlsson, R L Cohen, W C Hsiang, J D S Jones, editors), Math. Sci. Res. Inst. Publ. 27, Springer, New York (1994) 15-55 MR1268186

[6] M C Crabb, Fibrewise homology, Glasg. Math. J. 43 (2001) 199-208 MR1838625

[7] M C Crabb, W A Sutherland, Counting homotopy types of gauge groups, Proc. London Math. Soc. (3) 81 (2000) 747-768 MR1781154

[8] S K Donaldson, Connections, cohomology and the intersection forms of 4-manifolds, J. Differential Geom. 24 (1986) 275-341 MR868974

[9] Y Félix, J Oprea, Rational homotopy of gauge groups, Proc. Amer. Math. Soc. 137 (2009) 1519-1527 MR2465678

[10] EM Friedlander, Exceptional isogenies and the classifying spaces of simple Lie groups, Ann. Math. (2) 101 (1975) 510-520 MR0391078

[11] D H Gottlieb, Applications of bundle map theory, Trans. Amer. Math. Soc. 171 (1972) 23-50 MR0309111 
[12] B Gray, Homotopy commutativity and the EHP sequence, from: "Algebraic topology (Evanston, IL, 1988)”, (M Mahowald, S Priddy, editors), Contemp. Math. 96, Amer. Math. Soc. (1989) 181-188 MR1022680

[13] J Grbić, S D Theriault, Odd primary self-maps of low rank Lie groups, Canad. J. Math 62 (2010) 284-304

[14] H Hamanaka, A Kono, Unstable $K^{1}$-group and homotopy type of certain gauge groups, Proc. Roy. Soc. Edinburgh Sect. A 136 (2006) 149-155 MR2217512

[15] B Harris, On the homotopy groups of the classical groups, Ann. of Math. (2) 74 (1961) 407-413 MR0131278

[16] P Hilton, G Mislin, J Roitberg, Localization of nilpotent groups and spaces, North-Holland Math. Studies 15, North-Holland Publishing Co., Amsterdam (1975) MR0478146

[17] I M James, The topology of Stiefel manifolds, London Math. Soc. Lecture Note Ser. 24, Cambridge Univ. Press (1976) MR0431239

[18] D Kishimoto, A Kono, Note on mod p decompositions of gauge groups, Proc. Japan Acad. Ser. A 86 (2010) 15-17

[19] A Kono, A note on the homotopy type of certain gauge groups, Proc. Roy. Soc. Edinburgh Sect. A 117 (1991) 295-297 MR1103296

[20] A Kono, S Tsukuda, A remark on the homotopy type of certain gauge groups, J. Math. Kyoto Univ. 36 (1996) 115-121 MR1381542

[21] G E Lang, Jr, The evaluation map and EHP sequences, Pacific J. Math. 44 (1973) 201-210 MR0341484

[22] G Masbaum, On the cohomology of the classifying space of the gauge group over some 4-complexes, Bull. Soc. Math. France 119 (1991) 1-31 MR1101938

[23] M Mimura, G Nishida, H Toda, Mod p decomposition of compact Lie groups, Publ. Res. Inst. Math. Sci. 13 (1977) 627-680 MR0478187

[24] M Mimura, H Toda, Cohomology operations and homotopy of compact Lie groups. I, Topology 9 (1970) 317-336 MR0266237

[25] W A Sutherland, Function spaces related to gauge groups, Proc. Roy. Soc. Edinburgh Sect. A 121 (1992) 185-190 MR1169902

[26] S Terzić, The rational topology of gauge groups and of spaces of connections, Compos. Math. 141 (2005) 262-270 MR2099779

[27] S D Theriault, The $H$-structure of low-rank torsion free $H$-spaces, Q. J. Math. 56 (2005) 403-415 MR2161254

[28] S D Theriault, The odd primary $H$-structure of low rank Lie groups and its application to exponents, Trans. Amer. Math. Soc. 359 (2007) 4511-4535 MR2309196 
[29] H Toda, A topological proof of theorems of Bott and Borel-Hirzebruch for homotopy groups of unitary groups, Mem. Coll. Sci. Univ. Kyoto. Ser. A. Math. 32 (1959) 103-119 MR0108790

[30] H Toda, On iterated suspensions. I, II, J. Math. Kyoto Univ. 5 (1966) 87-142, 209-250 MR0210130

Department of Mathematical Sciences, University of Aberdeen Aberdeen AB24 3UE, United Kingdom

s.theriault@abdn.ac.uk

http://www.maths.abdn.ac.uk/ stephen/

Received: 23 July 2009 Revised: 10 December 2009 